\title{
Sz.-Nagy-Foias Theory and Lax-Phillips Type Semigroups in the Description of Quantum Mechanical Resonances
}

\author{
Y. Strauss* \\ Preprint No. 12 \\ $2003 / 2004$
}

May 11, 2004

\begin{abstract}
A quantum mechanical version of the Lax-Phillips scattering theory was recently developed. This theory is a natural framework for the description of quantum unstable systems. However, since the spectrum of the generator of evolution in this theory is unbounded from below, the existing framework does not apply to a large class of quantum mechanical scattering problems. It is shown in this work that the fundamental mathematical structure underlying the Lax-Phillips theory, i.e., the Sz.-Nagy-Foias theory of contraction operators on Hilbert space, can be used for the construction of a formalism in which models associated with a semibounded spectrum may be accomodated.
\end{abstract}

\section{Introduction}

The Lax-Phillips scattering theory [LP] was originally devised for the analysis of the scattering of electromagnetic or acoustic waves off compact obstacles; it is most sutible for handling hyperbolic wave equations in which the generator of evolution, or wave group, naturally has a continuous specrum on the whole real line. More recently Sjöstrand and Zworski [SZ1] extended

${ }^{*}$ Research supported by THE ISRAEL SCIENCE FOUNDATION (grant no. 188/02) and the Edmund Landau Center for Research in Mathematical Analysis and Related areas, sponsored by the Minerva Foundation (Germany). 
the scope of the Lax-Phillips theory to include more general cases of semibounded, compactly supported, perturbations of the Laplacian in the wave equation. The extended version of the theory plays a role in the ongoing effort in recent years to obtain various upper and lower bounds on the number of resonances (see [SZ1, PV] and references therein). The Lax-Phillips theory also plays an important role in geometric analysis where certain problems can be naturally formulated as scattering problems (see $[\mathrm{Z}]$ and references therein).

The description of resonances in the framework of the Lax-Phillips theory possess certain properties which may be considered as defining properties of an appropriate description of these objects. Resonances are identified according to their evolution in time under the action of a dynamical semigroup, i.e., the Lax-Phillips semigroup. Specifically, resonances are identified as eigenvalues of the generator of the Lax-Phillips semigroup. In fact, Sjöstrand and Sworski prove in [SZ1] that in the case of the Lax-Phillips theory, the eigenvalues of the generator of the Lax-Phillips semigroup coincide with the generally accepted identification of resonances with poles of (a generalized form of) the resolvent.

Many recent studies indicate that resonances should formaly be treated in the same way as eigenvalues (see [NSZ, Z] and references therein). Taking the analogy a step forward it is natural to ask whether it is possible to show that resonances are indeed eigenvalues of an operator closely related to the generator of evolution. This is known to be the case in the procedure of complex scaling (introduced in [AC, BC]; see also [HeSj, Hun, Sim1, Sim2, SZ2] and [HiSi] and references therein) and the method of rigged Hilbert space (see for example [B, BS, HoSi, PGS, BG]). For a recent abstract treatment of the problem of resonances along these lines see $[\mathrm{A}]$. The structure of the Lax-Phillips theory is again very appealing in this respect since the LaxPhillips semigroup is just the projection of the evolution into a subspace of the Hilbert space for the scattering problem.

If we require that the description of resonances would have the properties mentioned above i.e.:

(I) The time evolution of resonances is given in terms of a continuous, one parameter, contractive semigroup

$$
\mathbf{Z}\left(t_{1}\right) \mathbf{Z}\left(t_{2}\right)=\mathbf{Z}\left(t_{1}+t_{2}\right), \quad t_{1}, t_{2} \geq 0
$$

(II) A resonance is assigned, for each time $t$, a pure state in a Hilbert space.

then for most scattering problems in quantum mechanics the situation is not as favorable as in the Lax-Phillips theory. In fact, the standard treatment of 
the time evolution of resonances through the notion of the survival amplitude does not generically yield a semigroup law of evolution even in the pole approximation in which the propagator is approximated by taking only the contributions coming from the scattering poles. This fact becomes evident in the case of multiple scattering poles where the residues of the resolvent at different points in the complex plane are not orthogonal to each other and generate interference effects which destroy the semigroup property [HM]. In particular, it is interesting to relate the statements made here to some recent efforts to obtain resonance expansions of propagators [NSZ] and to recent work on time dependent theory of resonances [MS, SW, CS].

There has been some recent work on a quantum mechanical adaptation of the Lax-Phillips theory. Some progress has been made in [FP, HP, EH] and in [SHE] a general formalism was developed which was subsequently applied to certain simple Lee-Friedrichs type models in relativistic quantum field theory [SH1, SH2]. More recently, the general formalism developed in [SHE] has been used for the analysis of the Stark effect (Ben Ari T. and Horwitz L.P.: Stark effect in the Lax-Phillips scattering theory; in preparation). However, the formalism developed in [SHE] essentially retains the original structure of the Lax-Phillips theory and hence it is not sutible for the analysis of a large class of quantum mechanical problems, since the generator of evolution is required to have an unbounded spectrum from below as well as from above. A natural question then arises whether this situation can be amended. One of the goals of this paper is to show that, in a sense, the answer to this question is in the affirmative. More specifically, it is shown that the fundamental mathematical theory underlying the LaxPhillips structure, i.e., the Sz.-Nagy-Foias theory of contraction operators on Hilbert space $[\mathrm{NF}]$, can be used in the description of resonances for problems for which the generator of evolution has a semibounded spectrum.

The purpose of this paper is two fold. First, it is shown in Section 2 below that a formalism analogous to that of the Lax-Phillips scattring theory may be constructed for the description of resonances in scattrering problems for which the generator of evolution has a semibounded spectrum. Thus, two "representations" of the problem are defined, analogous to the LaxPhillips translation representations, in which the evolution of the scattering system is transformed into the analogue of translation, i.e., into a translation semigroup of Toeplitz operators on Hardy space. It is also possible to define an $S$-matrix, analogous to the Lax-Phillips $S$-matrix, mapping the incoming "representation" onto the outgoing "representation". Beyond mere analogy, in the formalism developed in Section 2 scattering resonances are defined exactly as in the Lax-Phillips theory, i.e., as eigenvalues of the generator of 
a Lax-Phillips type semigroup.

The second goal of this paper is to suggest that the tool box of methods available for the analysis of resonance phenomena should include the Sz.-Nagy-Foias theory of contraction operators on Hilbert space and, more generally, the part of operator theory dealing with model operators and decompositions $[\mathrm{K}]$. The Sz.-Nagy-Foias theory of contractions is an important part of the subject of model operators. Let $\mathcal{B}(\mathcal{H})$ be the space of bounded linear operators on a Hilbert space $\mathcal{H}$. An operator is a model operator for a class of operators in $\mathcal{B}(\mathcal{H})$ if every operator in that class is similar to a multiple of a part of it (a part of an operator is defined to be the restriction of the operator to an invariant subspace). In the case of contractions on Hilbert space the model operator is the backward shift (see [RR, K]). If a resonance is characterized by conditions (I) and (II) above it is then quite natural that its description will eventually be given in terms of the model for contractions, i.e, essentially by the restriction of the backward shift to an invariant subspace. Taking this point of view, the role of the Sz.-Nagy-Foias theory in the structures developed in Section 2 is not surprising and in fact unavoidable.

The rest of this paper is organized as follows: The Lax-Phillips scattering theory is briefly reviewed in Subsection 1.1. Subsection 1.2 provides a self contained, but rather restricted in scope, exposition of the parts of the Sz.-Nagy-Foias theory which are relevant to the rest of the paper. The Sz.-Nagy-Foias theory is thoroughly discussed in [NF]. Subsection 1.2 also describes the way that the Lax-Phillips semigroup can be naturally understood in the context of the Sz-Nagy-Foias theory. Section 2 contains the main results in this paper. After a preliminary discussion in Subsection 2.1, concerning Hilbert space nesting, a formalism enabling the use of the Sz.-Nagy-Foias theory for the description of resonances is developed in Subsection 2.2. Section 3 contains some comments on the framework introduced in Section 2 and concluding remarks are found in Section 4.

\subsection{The Lax-Phillips scattering theory}

This subsection provides a brief review of the Lax-Phillips scattering theory and establishes some of the notation which is used throughout the rest of this paper.

Consider a Hilbert space $\mathcal{H}$ and an evolution group of unitary operators $\{\mathbf{U}(t)\}_{t \in \mathbb{R}}$ on $\mathcal{H}$. Suppose that there exists two distinguished subspaces $\mathcal{D}_{-}$ 
and $\mathcal{D}_{+}$which have the properties that $\mathcal{D}_{-}$is orthogonal to $\mathcal{D}_{+}$and:

$$
\begin{aligned}
\mathbf{U}(t) \mathcal{D}_{-} & \subset \mathcal{D}_{-}, & & t \leq 0 \\
\mathbf{U}(t) \mathcal{D}_{+} & \subset \mathcal{D}_{+}, & & t \geq 0 \\
\cap_{t} \mathbf{U}(t) \mathcal{D}_{ \pm} & =\{0\} & & \\
\cup_{t} \mathbf{U}(t) \mathcal{D}_{ \pm} & =\mathcal{H} . & &
\end{aligned}
$$

We call $\mathcal{D}_{-}$the incoming subspace and $\mathcal{D}_{+}$the outgoing subspace. $\mathcal{D}_{-}$corresponds to incoming waves which do not interact with the target prior to $t=0$ and $\mathcal{D}_{+}$corresponds to outgoing waves which do not interact with the target after $t=0$. These properties are reflected in the stability properties of $\mathcal{D}_{-}$and $\mathcal{D}_{+}$in Eq. (1.2).

Let $L_{\mathcal{N}}^{2}(\mathbb{R})$ be the space of $L^{2}$ functions defined on $\mathbb{R}$ and taking their values in a Hilbert space $\mathcal{N}$. Ya. G. Sinai [CFS] proved the following theorem:

Theorem 1.1 (Ya. G. Sinai) if $\mathcal{D}_{+}$is an outgoing subspace with respect to a group of unitary operators $\mathbf{U}(t)$, then the Hilbert space $\mathcal{H}$ can be represented isometrically as the Hilbert space of functions $L_{\mathcal{N}}^{2}(\mathbb{R})$ for some auxiliary Hilbert space $\mathcal{N}$ so that $\mathbf{U}(t)$ goes into translation to the right by $t$ units, and $\mathcal{D}_{+}$is mapped onto $L_{\mathcal{N}}^{2}\left(\mathbb{R}^{+}\right)$. This representation is unique up to an isomorphism of $\mathcal{N}$.

A representation of this kind is called outgoing translation representation. An analogous representation theorem hold for an incoming subspace $\mathcal{D}_{-}$, i.e, there is a representation in which $\mathcal{H}$ is mapped onto the Hilbert space $L_{\mathcal{N}}^{2}(\mathbb{R})$, $\mathcal{D}_{-}$is mapped onto $L_{\mathcal{N}}^{2}\left(\mathbb{R}^{-}\right)$and $\mathbf{U}(t)$ acts as translation to the right by $t$ units. This representation is called the incoming translation representation.

For most purposes it is more convenient not to work with the translation representations but with two different representations, called spectral representations. By Fourier transformation of the incoming translation representation and the outgoing translation representation we obtain the incoming spectral representation and outgoing spectral representation respectively. According to the Paley-Wiener theorem [PW] in the incoming spectral representation, the subspace $\mathcal{D}_{-}$is represented by the Hilbert space of functions $H_{\mathcal{N}}^{+}(\mathbb{R})$ consisting of boundary values on $\mathbb{R}$ of functions in the Hardy space $H_{\mathcal{N}}^{2}(\Pi)$. Denoting the upper half of the complex plane by $\Pi$, the space $H_{\mathcal{N}}^{2}(\Pi)$ is characterized as the space of analytic vector valued functions on $\Pi$, taking their values in the auxiliary Hilbert space $\mathcal{N}$, and such that for 
any function $f \in H_{\mathcal{N}}^{2}(\Pi)$ we have

$$
\sup _{y>0} \int_{-\infty}^{+\infty}\|f(x+i y)\|_{\mathcal{N}}^{2} d x<C
$$

for some constant $C>0$. In the outgoing spectral representation the subspace $\mathcal{D}_{+}$is represented, according to the Paley-Wiener theorem, by the function space $H_{\mathcal{N}}^{-}(\mathbb{R})$ consisting of boundary values of functions in $H_{\mathcal{N}}^{2}(\bar{\Pi})$, a Hardy space of vector valued functions (taking values in $\mathcal{N}$ ) on the lower half-plane $\bar{\Pi}$. In the case of scalar valued functions used in the sequel in Section 2 below the same notation will be used but the subscript $\mathcal{N}$ for the auxiliary Hilbert space is omitted.

Let $\mathbf{W}_{+}$and $\mathbf{W}_{-}$denote the operators that map elements of $\mathcal{H}$ to their outgoing, respectively incoming, translation representers. We call the operator

$$
\mathbf{S}^{L . P} \equiv \mathbf{W}_{+} \mathbf{W}_{-}^{-1}
$$

the abstract scattering operator associated with the group $\mathbf{U}(t)$ and the pair of spaces $\mathcal{D}_{-}$and $\mathcal{D}_{+}$. It was proved by Lax and Phillips that $\mathbf{S}^{L . P}$ is equivalent to the standard definition of the scattering operator. The abstract scattering operator has the following properties:

a) $\mathbf{S}^{L . P}$ is unitary.

b) $\mathbf{S}^{L . P}$ commutes with translations

c) $\mathbf{S}^{L . P}$ maps $L_{\mathcal{N}}^{2}\left(\mathbb{R}^{-}\right)$into itself.

Property $(b)$ is due to the fact that $\mathbf{S}^{L . P .}$ is a map between two translation representations. One can understand property $(c)$ by noting that in the incoming translation representation the subspace $\mathcal{D}_{-}$is identified with the space of functions $L_{\mathcal{N}}^{2}\left(\mathbb{R}^{-}\right)$and in the outgoing translation representation $\mathcal{D}_{+}$is represented as $L_{\mathcal{N}}^{2}\left(\mathbb{R}^{+}\right)$. The orthogonality of $\mathcal{D}_{-}$and $\mathcal{D}_{+}$then implies that in the outgoing translation representation $\mathcal{D}_{-}$is represented by a subspace of $L_{\mathcal{N}}^{2}\left(\mathbb{R}^{-}\right)$and property $(c)$ above follows.

Going over to the spectral representations the scattering operator transforms into $\mathcal{S}^{L . P} \equiv \mathbf{F S}^{L . P} \mathbf{F}^{-1}$, where $\mathbf{F}$ is the Fourier transform operator. Properties $(a)-(c)$ above then imply corresponding properties for $\mathcal{S}^{L . P}$ :

a') $\mathcal{S}^{L . P .}$ is unitary.

b') $\mathcal{S}^{\text {L.P. }}$ commutes with multiplication by scalar functions. 
c') $\mathcal{S}^{L . P .}$ maps $H_{\mathcal{N}}^{+}(\mathbb{R})$ into itself.

According to a special case of a theorem of Foures and Segal [FS], an operator satisfying properties $\left(a^{\prime}\right)-\left(c^{\prime}\right)$ can be realized as a multiplicative, operator valued function $\mathcal{S}$, such that $\mathcal{S}(\sigma)$ maps $\mathcal{N}$ into $\mathcal{N}$ for each $\sigma \in \mathbb{R}$. This operator valued function satisfies:

a") $\mathcal{S}(\sigma)$ is the boundary value of an oprator valued function $\mathcal{S}(z)$ analytic for $\operatorname{Im} z>0$.

b") $\|\mathcal{S}(z)\|_{\mathcal{N}} \leq 1$ for $\operatorname{Im} z>0$

c") $\mathcal{S}(\sigma), \sigma \in \mathbb{R}$ is, pointwise, a unitary operator on the auxiliary Hilbert space $\mathcal{N}$.

Consider the family $\{\mathbf{Z}(t)\}_{t \geq 0}$ of operators on $\mathcal{H}$ defined by

$$
\mathbf{Z}(t) \equiv \mathbf{P}_{+} \mathbf{U}(t) \mathbf{P}_{-}, \quad(t \geq 0) .
$$

Here $\mathbf{P}_{+}$is the orthogonal projection of $\mathcal{H}$ onto the orthogonal complement of $\mathcal{D}_{+}$and $\mathbf{P}_{-}$is the orthogonal projection of $\mathcal{H}$ onto the orthogonal complement of $\mathcal{D}_{-}$. Lax and Phillips prove the following theorem

Theorem 1.2 The operators $\{\mathbf{Z}(t)\}_{t \geq 0}$ annihilate $\mathcal{D}_{+}$and $\mathcal{D}_{-}$, map the orthogonal complement $\mathcal{K}=\mathcal{H} \ominus\left(\mathcal{D}_{-} \oplus \mathcal{D}_{+}\right)$into itself, and form a strongly continuous semigroup (i.e. $\mathbf{Z}\left(t_{1}\right) \mathbf{Z}\left(t_{2}\right)=\mathbf{Z}\left(t_{1}+t_{2}\right)$ ) of contraction operators on $\mathcal{K}$. Furthermore, $\mathbf{Z}(t)$ tends strongly to zero as $t \rightarrow \infty: s-\lim _{t \rightarrow \infty} \mathbf{Z}(t) x=0$ for every $x$ in $\mathcal{K}$.

The family of operators $\{\mathbf{Z}(t)\}_{t \geq 0}$ is called the Lax-Phillips semigroup. The operator valued function $\mathcal{S}$, with properties $(a ")-\left(c^{\prime \prime}\right)$ above, is called the Lax-Phillips $S$-matrix. Properties $(a ")-(c ")$ characterize the LaxPhillips $S$-matrix as an analytic function on the upper half-plane. The analytic continuation of $\mathcal{S}$ to the lower half-plane is given by

$$
\mathcal{S}(z) \equiv\left[\mathcal{S}^{*}(\bar{z})\right]^{-1}, \quad \operatorname{Im} z<0 .
$$

One of the main results of the Lax-Phillips scattering theory is the following theorem

Theorem 1.3 Let $\mathbf{B}$ denote the generator of the semigroup $\mathbf{Z}(t)$. If $\operatorname{Im} \mu<$ 0 , then $\mu$ belongs to the point spectrum of $\mathbf{B}$ if and only if $\mathcal{S}^{*}(\bar{\mu})$ has a non-trivial null space.

This theorem establishes a very important relation between the eigenvalues of the generator $\mathbf{B}$ of the Lax-Phillips semigroup and zeros of the Lax-Phillips $S$-matrix $\mathcal{S}$ in the upper half-plane or poles of the analytic continuation of $\mathcal{S}$ to the lower half-plane. 


\subsection{Lax-Phillips theory and the Sz.-Nagy-Foias theory of contractions}

In this subsection the structure of the Lax-Phillips theory is put into the context of the more general theory of B. Sz.-Nagy and C. Foias concerning contraction operators on Hilbert space $[\mathrm{NF}]$.

Consider the Lax-Phillips semigroup $\{\mathbf{Z}(t)\}_{t \geq 0}$. According to Theorem 1.2 this semigroup acts non-trivially on the subspace $\mathcal{K}=\mathcal{H} \ominus\left(\mathcal{D}_{-} \oplus \mathcal{D}_{+}\right)$. For a vector $f \in \mathcal{K}$ and for $t_{1}, t_{2} \geq 0$ we have

$$
\begin{aligned}
\mathbf{Z}\left(t_{1}\right) \mathbf{Z}\left(t_{2}\right) f=\mathbf{P}_{+} \mathbf{U}\left(t_{1}\right) \mathbf{P}_{-} \mathbf{Z}\left(t_{2}\right) f=\mathbf{P}_{+} \mathbf{U}\left(t_{1}\right) \mathbf{Z}\left(t_{2}\right) f= \\
=\mathbf{P}_{+} \mathbf{U}\left(t_{1}\right) \mathbf{P}_{+} \mathbf{U}\left(t_{2}\right) f .
\end{aligned}
$$

The stability properties of the subspace $\mathcal{D}_{+}$(Eq. (1.2)) imply the following identity

$$
\mathbf{P}_{+} \mathbf{U}(t)\left(\mathbf{I}-\mathbf{P}_{+}\right)=0, \quad t \geq 0 .
$$

Inserting this identity into the r.h.s. of the previous equation we find, for $f \in \mathcal{K} \oplus \mathcal{D}_{-}$and $t_{1}, t_{2} \geq 0$

$$
\begin{aligned}
\mathbf{P}_{+} \mathbf{U}\left(t_{1}\right) \mathbf{P}_{+} \mathbf{U}\left(t_{2}\right) f=\mathbf{P}_{+} \mathbf{U}\left(t_{1}\right)\left[\left(\mathbf{I}-\mathbf{P}_{+}\right)+\mathbf{P}_{+}\right] \mathbf{U}\left(t_{2}\right) f= \\
\quad=\mathbf{P}_{+} \mathbf{U}\left(t_{1}\right) \mathbf{U}\left(t_{2}\right) f=\mathbf{P}_{+} \mathbf{U}\left(t_{1}+t_{2}\right) f .
\end{aligned}
$$

We note that, since for $f \in \mathcal{K}$ we have $\mathbf{P}_{+} \mathbf{U}\left(t_{1}+t_{2}\right) f=\mathbf{P}_{+} \mathbf{U}\left(t_{1}+t_{2}\right) \mathbf{P}_{-} f=$ $\mathbf{Z}\left(t_{1}+t_{2}\right) f$, Eq. (1.8) implies the semigroup law. Given a Lax-Phillips structure with an evolution group $\mathbf{U}(t)$ on the Hilbert space $\mathcal{H}$, consider the family of operators $\{\mathbf{T}(t)\}_{t \geq 0}$ such that $\mathbf{T}(t): \mathcal{H} \mapsto \mathcal{H}, t \geq 0$ and

$$
\mathbf{T}(t) \equiv \mathbf{P}_{+} \mathbf{U}(t), \quad t \geq 0 .
$$

Each element of this family of operators annihilates the subspace $\mathcal{D}_{+}$, as can be seen, for example, from Eq. (1.7). By Eq. (1.8) we find that for any vector $f \in \mathcal{D}_{-} \oplus \mathcal{K}$ we have

$$
\mathbf{T}\left(t_{1}\right) \mathbf{T}\left(t_{2}\right) f=\mathbf{T}\left(t_{1}+t_{2}\right) f, \quad t_{1}, t_{2} \geq 0
$$

and so the family $\{\mathbf{T}(t)\}_{t \geq 0}$ forms a one parameter continuous semigroup. We observe from Eq. (1.6) that, for $f \in \mathcal{K}$, we have

$$
\mathbf{Z}\left(t_{1}\right) \mathbf{Z}\left(t_{2}\right) f=\mathbf{T}\left(t_{1}\right) \mathbf{T}\left(t_{2}\right) f, \quad t_{1}, t_{2} \geq 0, f \in \mathcal{K} .
$$

Consider now the Lax-Phillips outgoing translation representation. Denote the outgoing translation representer of an operator $\mathbf{T}(t)$ from the family 
defined in Eq. (1.9) by $\tilde{\mathbf{T}}(t)$. Given any vector valued function $f \in L_{\mathcal{N}}^{2}(\mathbb{R})$ in the outgoing translation representation we have

$$
(\tilde{\mathbf{T}}(t) f)(s)=\left\{\begin{array}{ll}
f(s-t) & s \leq 0, \\
0 & s>0 .
\end{array}, \quad t \geq 0 .\right.
$$

Denote the generator of the semigroup $\{\mathbf{T}(t)\}_{t \geq 0}$ by $\mathbf{A}$. It is easy to show that the spectrum of $\mathbf{A}$ is $\sigma(\mathbf{A})=\{\mu \mid \operatorname{Im} \mu<0\}$. Denoting the outgoing translation representer of $\mathbf{A}$ by $\tilde{\mathbf{A}}$, the eigenfunctions of $\tilde{\mathbf{A}}$ are given by

$$
f_{\mu, \mathrm{n}}(s)=\left\{\begin{array}{ll}
e^{i \mu s} \mathrm{n} & s \leq 0, \\
0 & s>0 .
\end{array}, \quad \forall \mu \quad \operatorname{Im} \mu<0, \forall \mathrm{n} \in \mathcal{N}\right.
$$

where $f_{\mu, \mathrm{n}}$ is an eigenfunction of $\tilde{\mathbf{A}}$ with eigenvalue $\mu$.

As mentioned above one usually works in the spectral representations rather than in the translation representations. Hence, we would like to find the representation of the semigroup $\{\mathbf{T}(t)\}_{t \geq 0}$ and the eigenfunctions $f_{\mu, \mathrm{n}}$ in the outgoing spectral representation. For this we need the definition of a Toeplitz operator on the Hardy space $H_{\mathcal{N}}^{+}(\mathbb{R})$ (see for example $[R R]$ and references therein):

Definition (Toeplitz operator on $H_{\mathcal{N}}^{+}(\mathbb{R})$ ): Let $W \in L_{\mathcal{B}(\mathcal{N})}^{\infty}(\mathbb{R})$ $(\mathcal{B}(\mathcal{N})$ is the space of bounded linear operators on $\mathcal{N})$. Let $P_{+}$denote the projection of $L_{\mathcal{N}}^{2}(\mathbb{R})$ on $H_{\mathcal{N}}^{+}(\mathbb{R})$. The operator $T_{W}: H_{\mathcal{N}}^{+}(\mathbb{R}) \rightarrow H_{\mathcal{N}}^{+}(\mathbb{R})$ defined by

$$
T_{W} f=P_{+} W f, \quad f \in H_{\mathcal{N}}^{+}(\mathbb{R})
$$

is called a Toeplitz operator $\left(\right.$ on $\left.H_{\mathcal{N}}^{+}(\mathbb{R})\right)$ with symbol $W$. Here $W f$ is the operator of pointwise application of $W$ to $f$ i.e., $(W f)(\sigma)=W(\sigma) f(\sigma)$, $\sigma \in \mathbb{R}$.

Define $\{u(t)\}_{t \in \mathbb{R}}$, a family of multiplicative operators on $L_{\mathcal{N}}^{2}(\mathbb{R})$, by

$$
[u(t) f](\sigma)=e^{-i \sigma t} f(\sigma), \quad f \in L_{\mathcal{N}}^{2}(\mathbb{R}), \sigma \in \mathbb{R} .
$$

Denote by $\hat{\mathbf{T}}(t)$ the outgoing spectral representer of the semigroup element $\mathbf{T}(t)$. Taking the Fourier transform of Eq. (1.12) and using the definition of a Toeplitz operator we find that $\hat{\mathbf{T}}(t)$ is given by

$$
\hat{\mathbf{T}}(t) f=P_{+} u(t) f=T_{u(t)} f \quad f \in H_{N}^{+}(\mathbb{R}), t \geq 0
$$

and $\hat{\mathbf{T}}(t) f=0$ for $f \in H_{\mathcal{N}}^{-}(\mathbb{R})$. The semigroup $\{\mathbf{T}(t)\}_{t \geq 0}$ is, therefore, represented in the outgoing spectral representation, by the semigroup of 
Toeplitz operators on $H_{\mathcal{N}}^{+}(\mathbb{R})$ with symbols $u(t)$ (and a trivial action on $\left.H_{\mathcal{N}}^{-}(\mathbb{R})\right)$.

Define the map $\hat{\mathbf{W}}_{+}: \mathcal{H} \mapsto L_{\mathcal{N}}^{2}(\mathbb{R})$ by $\hat{\mathbf{W}}_{+} \equiv \mathbf{F} \mathbf{W}_{+}$(recall that $\mathbf{W}_{+}$ is the map of $\mathcal{H}$ onto the outgoing translation representation and $\mathbf{F}$ is the Fourier transform operator). Then $\hat{\mathbf{W}}_{+}$maps $\mathcal{H}$ onto the Lax-Phillips outgoing spectral representation and we can summarize the discussion above by the observation that for any $f \in \mathcal{K}$ we have

$$
\hat{\mathbf{W}}_{+} \mathbf{Z}(t) f=\hat{\mathbf{W}}_{+} \mathbf{T}(t) f=\hat{\mathbf{T}}(t) f_{\text {out }}=T_{u(t)} f_{\text {out }} \quad t \geq 0, f \in \mathcal{K}
$$

where $f_{\text {out }}=\hat{\mathbf{W}}_{+} f$ is the outgoing spectral representer of $f \in \mathcal{K}$. Put differently, letting $\hat{\mathbf{Z}}(t)=\hat{\mathbf{W}}_{+} \mathbf{Z}(t) \hat{\mathbf{W}}_{+}^{-1}$ be the outgoing spectral representer of $\mathbf{Z}(t)$ and letting $\hat{\mathcal{K}}=\hat{\mathbf{W}}_{+} \mathcal{K}$ be the subspace of $L_{\mathcal{N}}^{2}(\mathbb{R})$ representing $\mathcal{K}$ in the outgoing spectral representation, we have

$$
\hat{\mathbf{Z}}(t)=T_{u(t)} \mid \hat{\mathcal{K}} .
$$

Taking the Fourier transform of Eq. (1.13) we find that in the outgoing spectral representation the eigenfunctions of the generator $\hat{\mathbf{A}}$ of the semigroup $\{\hat{\mathbf{T}}(t)\}_{t \geq 0}=\left\{T_{u(t)}\right\}_{t \geq 0}$ are given by

$$
\hat{f}_{\mu, \mathrm{n}}(\sigma)=i \frac{\mathrm{n}}{\sigma-\mu} \quad \forall \mu \operatorname{Im} \mu<0, \forall \mathrm{n} \in \mathcal{N} .
$$

Eq. (1.17) and Eq. (1.18) raise the question of the characterization, out of all of the eigenvectors of the generator of the semigroup $\left\{T_{u(t)}\right\}_{t \geq 0}$ given in Eq. (1.18), of those which are contained in $\hat{\mathcal{K}}$. These will be the eigenvectors of the Lax-Phillips semigroup. The rest of this section deals with this question.

We start with the observation that following the discussion of the properties of the Lax-Phillips $S$-matrix in the previous subsection we know that the subspace $\hat{\mathcal{K}}$, representing $\mathcal{K} \in \mathcal{H}$ in the outgoing spectral representation, is given by

$$
\hat{\mathcal{K}}=H_{\mathcal{N}}^{+}(\mathbb{R}) \ominus \mathcal{S} H_{\mathcal{N}}^{+}(\mathbb{R})
$$

where $\mathcal{S}$ is an operator valued function with the properties (a")-(c") listed in the previous subsection. Such a function is identified as belonging to the class of operators on $H_{\mathcal{N}}^{+}(\Pi)$ known as inner functions. Thus we conclude that the representation of an element $\mathbf{Z}(t)$ of the Lax-Phillips semigroup in the outgoing spectral representation is always of the form

$$
\hat{\mathbf{Z}}(t)=T_{u(t)} \mid\left(H_{\mathcal{N}}^{+}(\mathbb{R}) \ominus \mathcal{S} H_{\mathcal{N}}^{+}(\mathbb{R})\right) \quad t \geq 0
$$


where $\mathcal{S}$ is some inner function. By a slight abuse of terminology a semigroup of the type of Eq. (1.20) will be called in the rest of this paper a Lax-Phillips semigroup. From Eq. (1.20) we can infer the general form of an element of the adjoint semigroup $\hat{\mathbf{Z}}^{*}(t)$. Using the notation of Eq. (1.19) we get

$$
\hat{\mathbf{Z}}^{*}(t)=P_{\hat{\mathcal{K}}} T_{u(t)}^{*}\left|\hat{\mathcal{K}}=P_{\hat{\mathcal{K}}} T_{\bar{u}(t)}\right| \hat{\mathcal{K}} \quad t \geq 0 .
$$

In order to understand the structure described here and the origin of Theorem 1.3, we turn to the Sz.-Nagy-Foias theory of contraction operators on Hilbert space $[\mathrm{NF}]$. We start with the observation that the family $\left\{T_{u(t)}\right\}_{t \geq 0}$ is a continuous semigroup of contraction operators and the adjoint family $\left\{T_{u(t)}^{*}\right\}_{t \geq 0}=\left\{T_{\bar{u}(t)}\right\}_{t \geq 0}$ is also a semigroup of contractions. Moreover, $\left\{T_{u(t)}^{*}\right\}_{t \geq 0}$ is an isometric semigroup. It is a basic result of the Sz.-NagyFoias theory that the study of continuous semigroups of contractions can be reduced to the study of a single contraction operator. If $\{T(t)\}_{t \geq 0}$ is a one parameter continuous semigroup of contractions with generator $A$ defined by

$$
A h=\lim _{t \rightarrow 0^{+}} \frac{1}{t}(T(t)-I) h
$$

then the study of $\{T(t)\}_{t \geq 0}$ reduces to the study of its cogenerator $T$ defined by

$$
T=\frac{A+I}{A-I}
$$

It is easy to show that the cogenerator $T$ is a contraction. The elements of a semigroup $\{T(t)\}_{t \geq 0}$ are given in terms of the cogenerator $T$ by

$$
T(t)=\exp \left(t \frac{T+I}{T-I}\right) .
$$

Denoting the cogenerator of the semigroup $\left\{T_{\bar{u}(t)}\right\}_{t \geq 0}$ by $S_{T}: H_{\mathcal{N}}^{+}(\mathbb{R}) \rightarrow$ $H_{\mathcal{N}}^{+}(\mathbb{R})$, one finds that

$$
\left(S_{T} f\right)(\sigma)=\frac{\sigma-i}{\sigma+i} f(\sigma), \quad \sigma \in \mathbb{R}, f \in H_{\mathcal{N}}^{+}(\mathbb{R}) .
$$

The cogenerator of $\left\{T_{\bar{u}(t)}\right\}_{t \geq 0}$ is then identified as the canonical shift operator on $H_{\mathcal{N}}^{+}(\mathbb{R})$ (see for example $[R R]$ ). By Eq. (1.24) we then have

$$
T_{u(t)}^{*}=T_{\bar{u}(t)}=\exp \left(t \frac{S_{T}+I}{S_{T}-I}\right) .
$$


Using Eq. (1.21) we find that

$$
\hat{\mathbf{Z}}^{*}(t)=P_{\hat{\mathcal{K}}} \exp \left(t \frac{S_{T}+I}{S_{T}-I}\right) \mid \hat{\mathcal{K}} .
$$

Two other important ingredients of the Sz.-Nagy-Foias theory are the notions of unitary and isometric dilation of a contraction operator and the functional calculus for completely non unitary (c.n.u.) contractions. A dilation of an operator is defined as follows:

Definition (Dilation): Assume that for an operator $B$ on a Hilbert space $\mathcal{H}$ and an operator $A$ on a Hilbert space $\mathcal{H}^{\prime}$ the following relations hold

(i) $\mathcal{H}$ is a subspace of $\mathcal{H}^{\prime}$.

(ii) $B x=P_{\mathcal{H}} A x$ for all $x \in \mathcal{H}$, where $P_{\mathcal{H}}$ denotes the orthogonal projection from $\mathcal{H}^{\prime}$ into $\mathcal{H}$.

We call $A$ a dilation of $B$ if (i) and (ii) hold and if

$$
B^{n} x=P_{\mathcal{H}} A^{n} x \quad x \in \mathcal{H}, n=1,2,3, \ldots
$$

Sz.-Nagy and Foias prove ([NF], Chapter I) that for every contraction $T$ on a Hilbert space $\mathcal{H}$ there exists an isometric (and a unitary) dilation $V$ on some Hilbert space $\mathcal{R}$, which is moreover minimal in the sense that

$$
\mathcal{R}=\vee_{0}^{\infty} V^{n} \mathcal{H} .
$$

The minimal isometric dilation is determind up to an isomorphism. The space $\mathcal{H}$ is invariant for $V^{*}$ and we have

$$
T P_{\mathcal{H}}=P_{\mathcal{H}} V, \quad T^{*}=V^{*} \mid \mathcal{H}
$$

where $P_{\mathcal{H}}$ denotes the orthogonal projection from $\mathcal{R}$ onto $\mathcal{H}$.

Sz.-Nagy and Foias show ([NF], Chapter III) that the function space $H^{\infty}(D)$ ( $D$ is the open unit disk), regarded as an algebra with an involution under the usual addition and multiplication of functions, can be used to define a functional calculus for completely non-unitary (c.n.u.) contractions (for example see $[\mathrm{NF}]$ ). By this we mean that, for a c.n.u. contraction $T$ on a Hilbert space $\mathcal{H}$ there exists an algebra homomorphism of $H^{\infty}(D)$ into $B(\mathcal{H})$ (the space of bounded operators on $\mathcal{H}$ ). The map providing 
this homomorphism is defined, for a function $a \in H^{\infty}(D)$ having the series expansion $a(z)=\sum_{n=0}^{\infty} c_{n} z^{n}$, by

$$
a(z)=\sum_{n=0}^{\infty} c_{n} z^{n} \rightarrow a(T)=\lim _{r \rightarrow 1^{-}} a(r T)=\lim _{r \rightarrow 1^{-}}\left(\sum_{n=0}^{\infty} c_{n} r^{n} T^{n}\right) .
$$

Under this mapping we find, for a function $a \in H^{\infty}(D)$ and an isometric dilation $V$ of a c.n.u. contraction $T$, the following relation

$$
a(T) h=P_{\mathcal{H}} a(V) h \quad h \in \mathcal{H}, a \in H^{\infty}(D) .
$$

Define

$$
T_{\hat{\mathbf{Z}}^{*}} \equiv P_{\hat{\mathcal{K}}} S_{T} \mid \hat{\mathcal{K}}
$$

where $\hat{\mathcal{K}} \in H^{+}(\mathbb{R})$ defined in Eq. (1.19). The operator $P_{\hat{\mathcal{K}}} S_{T} \mid \hat{\mathcal{K}}$ is called a compression of the shift into the subspace $\hat{\mathcal{K}}$. It can be shown that, for any inner function $\mathcal{S}$, the shift $S_{T}$ is an isometric dilation of the compression of $S_{T}$ into $\hat{\mathcal{K}}$, i.e., it is an isometric dilation of $T_{\hat{\mathbf{Z}}^{*}}$. Since the function

$$
e_{t}(z) \equiv \exp \left(t \frac{z+1}{z-1}\right)
$$

is inner, Eq. (1.30) implies that

$$
\exp \left(t \frac{T_{\hat{\mathbf{Z}}^{*}}+I}{T_{\hat{\mathbf{Z}}^{*}}-I}\right)=P_{\hat{\mathcal{K}}} \exp \left(t \frac{S_{T}+I}{S_{T}-I}\right)\left|\hat{\mathcal{K}}=P_{\hat{\mathcal{K}}} T_{\bar{u}(t)}\right| \hat{\mathcal{K}}=\hat{\mathbf{Z}}^{*}(t) .
$$

The operator $T_{\hat{\mathbf{Z}}^{*}}$ is therefore the cogenerator of the semigroup $\left\{\hat{\mathbf{Z}}^{*}(t)\right\}_{t \geq 0}$. Indeed, given the family of functions $\phi_{t} \in H^{\infty}(D)$ defined by

$$
\phi_{t}(z)=\frac{z-1+t}{z-1-t} \quad t>0
$$

Sz.-Nagy and Foias prove ([NF], Chapter III, Theorem 8.1) that the cogenerator $T$ of a contractive semigroup $\{T(t)\}_{t \geq 0}$ is obtained by

$$
T=\lim _{t \rightarrow 0^{+}} \phi_{t}(T(t))
$$

and applying this relation to $\left\{\hat{\mathbf{Z}}^{*}(t)\right\}_{t \geq 0}$ we get

$$
\begin{array}{r}
\lim _{t \rightarrow 0^{+}} \phi_{t}\left(\hat{\mathbf{Z}}^{*}(t)\right)=\lim _{t \rightarrow 0^{+}} \phi_{t} \circ e_{t}\left(T_{\hat{\mathbf{Z}}^{*}(t)}\right)=\lim _{t \rightarrow 0^{+}} P_{\hat{\mathcal{K}}} \phi_{t} \circ e_{t}\left(S_{T}\right) \mid \hat{\mathcal{K}}= \\
=\lim _{t \rightarrow 0^{+}} P_{\hat{\mathcal{K}}} \phi_{t}(\bar{u}(t))\left|\hat{\mathcal{K}}=P_{\hat{\mathcal{K}}} S_{T}\right| \hat{\mathcal{K}}=T_{\hat{\mathbf{Z}}^{*}} .
\end{array}
$$


We conclude that the compression of the shift to the subspace $\hat{\mathcal{K}}$ is the cogenerator of the semigroup $\left\{\hat{\mathbf{Z}}^{*}(t)\right\}_{t \geq 0}$ and hence

$$
T_{\hat{\mathbf{Z}}^{*}}=P_{\hat{\mathcal{K}}} S_{T} \mid \hat{\mathcal{K}}=\frac{\hat{\mathbf{B}}^{*}+I}{\hat{\mathbf{B}}^{*}-I}
$$

where $\hat{\mathbf{B}}^{*}$ is the generator of the semigroup $\left\{\hat{\mathbf{Z}}^{*}(t)\right\}_{t \geq 0}$. We note also that, using Eq. (1.30), we obtain

$$
T_{\hat{\mathbf{Z}}}=\frac{\hat{\mathbf{B}}+I}{\hat{\mathbf{B}}-I}=S_{T}^{*} \mid \hat{\mathcal{K}}
$$

where $\hat{\mathbf{B}}$ is the generator and $T_{\hat{\mathbf{Z}}}$ is the cogenerator of the Lax-Phillips semigroup $\{\hat{\mathbf{Z}}(t)\}_{t \geq 0}$.

For a contraction $T$ on a Hilbert space $\mathcal{H}$ we define the defect operators $D_{T}$ and $D_{T^{*}}$ by

$$
D_{T} \equiv\left(I-T^{*} T\right)^{1 / 2}, \quad D_{T^{*}} \equiv\left(I-T T^{*}\right)^{1 / 2} .
$$

The defect spaces $\mathcal{D}_{T}$ and $\mathcal{D}_{T^{*}}$ are defind by

$$
\mathcal{D}_{T}=\overline{D_{T} \mathcal{H}}, \quad \mathcal{D}_{T^{*}}=\overline{D_{T^{*}} \mathcal{H}} .
$$

For every complex number $\lambda$ for which the operator $I-\lambda T^{*}$ is boundedly invertible, define

$$
\Theta_{T}(\lambda) \equiv\left[-T+\lambda D_{T^{*}}\left(I-\lambda T^{*}\right)^{-1} D_{T}\right] \mid \mathcal{D}_{T}
$$

the values of the operator valued function $\Theta_{T}(\lambda)$ are bounded operators from $\mathcal{D}_{T}$ into $\mathcal{D}_{T^{*}}$. When considered on the unit disk $D, \Theta_{T}(\lambda)$ is a purely contractive, analytic, operator valued function, i.e., it is a contraction from $\mathcal{D}_{T}$ into $\mathcal{D}_{T^{*}}$ for every $\lambda$ in the unit disk. The function $\Theta_{T}(\lambda)$ is called the characteristic function of the contraction $T$ (see $[\mathrm{NF}]$ Chapter VI). The main theorem of the Lax- Phillips scattering theory, relating eigenvalues of the generator of the Lax-Phillips semigroup and singularities of the Lax-Phillips $S$-matrix, is then a direct result of the the following theorem, proved by Sz.-Nagy and C. Foias ([NF] Chapter VI, Theorem 4.1):

Theorem 1.4 Let $T$ be a c.n.u. contraction on $\mathcal{H}$. Denote by $\alpha_{T}$ the set of points $\mu \in D$ for which the operator $\Theta_{T}(\mu)$ is not boundedly invertible, together with the points $\mu \in C$ not lying on any of the open arcs of $C$ on 
which $\Theta_{T}(\lambda)$ is a unitary operator valued analytic function of $\lambda$. Furthermore, denote by $\alpha_{T}^{0}$ the set of points $\mu \in D$ for which $\Theta_{T}(\lambda)$ is not invertible at all. Then

$$
\sigma(T)=\alpha_{T}
$$

and

$$
\sigma_{p}(T)=\alpha_{T}^{0}
$$

Given the cogenerator $T_{\hat{\mathbf{Z}}^{*}}$ we can define its characteristic function $\Theta_{T_{\hat{\mathbf{Z}}^{*}}}(\lambda)$ and obtain the spectrum of $T_{\hat{\mathbf{Z}}^{*}}$ in the closed unit disk through Theorem 1.4. In this particular case the function $\Theta_{T_{\hat{\mathbf{z}}^{*}}}$ is an inner function on the unit disk. We can now pass from the unit disk to the upper half-plane by setting

$$
\mathcal{S}(z)=\Theta_{T_{\hat{\mathbf{Z}}^{*}}}\left(\frac{z-i}{z+i}\right) \quad, \operatorname{Im} z>0 .
$$

The operator valued function $\mathcal{S}$ on the l.h.s. of Eq. (1.38) is exactly the inner function appearing in Eq. (1.19) or Eq. (1.20), defining the subspace $\hat{\mathcal{K}} \subset H_{\mathcal{N}}^{+}(\mathbb{R})$ and identical to the Lax-Phillips $S$-matrix. Theorem 1.4 above then immediately implies Theorem 1.3.

Recapitulating the discussion in this section, we have seen that $\hat{\mathbf{Z}}(t)$, the outgoing spectral representer of an element of the Lax-Phillips semigroup $\mathbf{Z}(t)$, is of the form of a restriction of a Toeplitz operator $T_{u(t)}$ to a subspace $\hat{\mathcal{K}} \subset H_{\mathcal{N}}^{+}(\mathbb{R})$ given by $\hat{\mathcal{K}}=H_{\mathcal{N}}^{+}(\mathbb{R}) \ominus \mathcal{S} H_{\mathcal{N}}^{+}(\mathbb{R})$, for some inner function $\mathcal{S}$. The Lax-Phillips $S$-matrix $\mathcal{S}$ is essentialy the characteristic function for the generator $\hat{\mathbf{B}}^{*}$ of the semigroup $\hat{\mathbf{Z}}^{*}(t)$. The cogenerator $T_{\hat{\mathbf{Z}}^{*}}$ of $\hat{\mathbf{Z}}^{*}(t)$ is identified as a compression of the shift and its characteristic function is an inner function. The correspondence between the points in $\Pi$ where $\mathcal{S}$ has a non-trivial null space (or poles of the analytic continuation of $\mathcal{S}$ to $\bar{\Pi}$ ) and eigenvalues of the generator of $\hat{\mathbf{Z}}(t)$ (or $\mathbf{Z}(t)$ ) then arises from a theorem in operator theory (namely Theorem 1.4 above) relating the points of singularity of the characteristic function for a contraction $T$ to the spectrum of $T$.

\section{Description of resonances}

\subsection{Preliminary discussion}

In this section the structures described in Subsection 1.2 are utilized for the description of quantum mechanical resonances. The structures developed 
below bear general resemblance to the structure of the Lax-Phillips scattering theory. For a given scattering problem an "incoming representation" and an "outgoing representation" are defined, as well as the "S-matrix" transforming between the two. The evolution goes in these "representations" into the analogue of translation, i.e., the semigroup of Toeplits operators $\left\{T_{u(t)}\right\}_{t \geq 0}$. Moreover, beyond general resemblance, the framework developed below makes explicit use of the fundamental structures of the Lax-Phillips theory originating from the Sz.-Nagy-Foias theory of contractions. Time evolution of resonances is actually described by a Lax-Phillips type semigroup of the form of Eq. (1.20) and the relation between eigenvalues corresponding to resonances and zeros of the $S$-matrix results from the Sz.-Nagy-Foias theory in the same way as described above in Subsection 1.2 .

The starting point for the developments in this section is a method, proposed by A. Grossmann [G], for the description of quantum mechanical resonances through the use of Hilbert space nesting.

Let $\mathcal{H}_{0}$ and $\mathcal{H}_{1}$ be (infinitely dimensional, separable) Hilbert spaces. A nesting map of $\mathcal{H}_{1}$ into $\mathcal{H}_{0}$ is a linear mapping $\theta$ such that:

1.) The domain of $\theta$ is $\mathcal{H}_{1}$ and $\theta$ is continuous on $\mathcal{H}_{1}$.

2.) The range of $\theta$ is dense in $\mathcal{H}_{0}$.

3.) $\theta$ is one to one.

The adjoint of $\theta$ is defined in the usual way by the relation

$$
(f, \theta g)_{\mathcal{H}_{0}}=\left(\theta^{*} f, g\right)_{\mathcal{H}_{1}}
$$

$\theta^{*}$ is a nesting map of $\mathcal{H}_{0}$ into $\mathcal{H}_{1}$. We note that the properties of the map $\theta$ puts it in the class of quasi-affine transforms between $\mathcal{H}_{1}$ and $\mathcal{H}_{0}$ (see, for example $[\mathrm{NF}, \mathrm{K}])$. A nested Hilbert space is defined as follows:

Definition (nested Hilbert space): A nested Hilbert space $\left(\mathcal{H}_{1}, \mathcal{H}_{0}, \theta\right)$ is defined to be a structure consisting of the two Hilbert spaces $\mathcal{H}_{0}$ and $\mathcal{H}_{1}$, a nesting map $\theta$ of $\mathcal{H}_{1}$ into $\mathcal{H}_{0}$ and the adjoint nesting map $\theta^{*}$.

Given a nested Hilbert space $\left(\mathcal{H}_{1}, \mathcal{H}_{0}, \theta\right)$ and an operator $\mathbf{A}$ on $\mathcal{H}_{0}$, Grossmann considered two types of operators, $\tilde{\mathbf{A}}$ and $\hat{\mathbf{A}}$ defined on $\mathcal{H}_{1}$. Respectively, these operators are defined to be

$$
\tilde{\mathbf{A}}=\theta^{*} \mathbf{A} \theta
$$


and

$$
\hat{\mathbf{A}}=\theta^{-1} \mathbf{A} \theta .
$$

We observe that the operator $\hat{\mathbf{A}}$ in Eq. (2.3) is well defined for any operator A which leaves the range of $\theta$ invariant. Let $f \in \mathcal{H}_{1}$ be in the domain of $\tilde{\mathbf{A}}$. For every $g \in \mathcal{H}_{1}$ we then have

$$
(g, \tilde{\mathbf{A}} f)_{\mathcal{H}_{1}}=\left(g, \theta^{*} \mathbf{A} \theta f\right)_{\mathcal{H}_{1}}=(\theta g, \mathbf{A} \theta f)_{\mathcal{H}_{0}} .
$$

We see that matrix elements of $\mathbf{A}$ on the dense set $\theta \mathcal{H}_{1} \subset \mathcal{H}_{0}$ can be calculated as matrix elements of $\tilde{\mathbf{A}}$ on $\mathcal{H}_{1}$. Put differently, the operator $\tilde{\mathbf{A}}$ on $\mathcal{H}_{1}$ may be defined through the knowledge of a subset of the matrix elements of $\mathbf{A}$ on $\mathcal{H}_{0}$. Moreover, if $\mathbf{A}$ is bounded and self-adjoint then $\tilde{\mathbf{A}}$ is also bounded and self-adjoint. Grossmann considers the resolvent $\mathbf{G}(z)=$ $(z-\mathbf{H})^{-1}$ of a scattering Hamiltonian $\mathbf{H}$ and defines through the nesting $\left(\mathcal{H}_{1}, \mathcal{H}_{0}, \theta\right)$ the operator $\tilde{\mathbf{G}}(z)$ according to Eq. (2.2). Since the matrix elements of $\tilde{\mathbf{G}}(z)$ between elements of $\mathcal{H}_{1}$ are a subset of the set of matrix elements of $\mathbf{G}(z)$ on $\mathcal{H}_{0}$, it may happen that $\tilde{\mathbf{G}}(z)$ has a larger domain of analyticity than $\mathbf{G}(z)$. In particular, the domain of analyticity of $\tilde{\mathbf{G}}(z)$ may cross the natural boundary of analyticity of $\mathbf{G}(z)$ along the positive real axis so that it may be possible to reach a second Riemann sheet resonance pole of the resolvent.

The matrix elements of $\hat{\mathbf{A}}$ are, on the other hand, generically different from those of $\mathbf{A}$ due to the different scalar product and, in general, $\hat{\mathbf{A}}$ is neither bounded nor symmetric. It is possible to show that the eigenvalues of $(\hat{\mathbf{A}})^{*}$ include the "improper" eigenvalues of $\mathbf{A}^{*}$.

Let $\mathbf{A}$ be a densely defined operator on $\mathcal{H}_{0}$ such that the operator $\left(\widehat{\mathbf{A}}^{*}\right)^{*}$ is also densely defined. A complex number $z_{0}$ is defined to be a generalized eigenvalue of $\mathbf{A}$ if there is an element $f \in \mathcal{H}_{1}$ such that

$$
\left(\widehat{\mathbf{A}}^{*}\right)^{*} f=z_{0} f .
$$

In particular, it is easy to show that eigenvalues of $\mathbf{A}$ are also eigenvalues of $\left(\widehat{\mathbf{A}}^{*}\right)^{*}$.

If in the Hilbert space nesting $\left(\mathcal{H}_{1}, \mathcal{H}_{0}, \theta\right)$ the map $\theta: \mathcal{H}_{1} \mapsto \mathcal{H}_{0}$ is a contraction then we have a contractive nesting of $\mathcal{H}_{1}$ into $\mathcal{H}_{0}$. This property of $\theta$ a priori adds more structure to the resulting framework. A short discussion of this point is found in Section 3 below.

\section{$2.2 \quad$ Main results}

In a similar way to the Lax-Phillips scattering theory, this work centers around three main results. The existence of appropriate incoming and out- 
going nesting structures, the existence of a continuous semigroup of contraction operators and the identification of the resonances of the theory, the eigenvalues of the generator of the semigroup, with zeros of the " $S$-matrix" mapping between the incoming and outgoing nesting structures.

Starting with the nesting structures, we have the following theorem:

Theorem 2.1 (outgoing contractive nesting) Let $\mathbf{H}_{0}$ and $\mathbf{H}$ be selfadjoint operators on a Hilbert space $\mathcal{H}$. Let $\{\mathbf{U}(t)\}_{t \in \mathbb{R}}$ be the unitary evolution group on $\mathcal{H}$ generated by $\mathbf{H}$ (i.e. $\mathbf{U}(t)=\exp (-i \mathbf{H} t)$ ). Denote by $\mathcal{H}_{a c}^{0}$ and $\mathcal{H}_{a c}$, respectively, the absolutely continuous subspaces of $\mathbf{H}_{0}$ and $\mathbf{H}$. Assume that the absolutely continuous spectrum of $\mathbf{H}_{0}$ and $\mathbf{H}$ has multiplicity one and that it satisfies $\sigma_{a c}\left(\mathbf{H}_{0}\right)=\sigma_{a c}(\mathbf{H})=\mathbb{R}^{+}$. Assume furthermore that the Møller wave operator $\boldsymbol{\Omega}^{-} \equiv \boldsymbol{\Omega}^{-}\left(\mathbf{H}_{0}, \mathbf{H}\right): \mathcal{H}_{a c}^{0} \mapsto \mathcal{H}_{a c}$ exists and is complete. Then there exists a map $\hat{\mathbf{\Omega}}_{+}: \mathcal{H}_{a c} \mapsto H^{+}(\mathbb{R})$ such that

$(\alpha)\left(\mathcal{H}_{a c}, H^{+}(\mathbb{R}), \hat{\boldsymbol{\Omega}}_{+}\right)$is a contractive Hilbert space nesting of $\mathcal{H}_{a c}$ into $H^{+}(\mathbb{R})$.

( $\beta$ ) For every $t \geq 0$ define the Grossmann type operator $\widehat{\mathbf{U}^{*}}(t): H^{+}(\mathbb{R}) \mapsto$ $H^{+}(\mathbb{R})$ according to $E q$. (2.3), i.e., $\widehat{\mathbf{U}}^{*}(t)=\left(\hat{\boldsymbol{\Omega}}_{+}^{*}\right)^{-1} \mathbf{U}^{*}(t) \hat{\mathbf{\Omega}}_{+}^{*}$. These operators are well defined and, for every $t \geq 0$ and every $f \in \mathcal{H}_{a c}$ we have

$$
\hat{\mathbf{\Omega}}_{+} \mathbf{U}(t) f=\left(\widehat{\mathbf{U}^{*}(t)}\right)^{*} \hat{\mathbf{\Omega}}_{+} f=T_{u(t)} f_{\text {out }} \quad t \geq 0
$$

where $f_{\text {out }}=\hat{\boldsymbol{\Omega}}_{+} f$ and $T_{u(t)}$ is the Toeplitz operator with symbol $u(t)$ given by Eq. (1.14).

The nesting $\left(\mathcal{H}_{a c}, H^{+}(\mathbb{R}), \hat{\boldsymbol{\Omega}}_{+}\right)$is referred to below as the outgoing contractive nesting of $\mathcal{H}_{a c}$.

\section{Remarks:}

1. It follows from Eq. (2.4) and the discussion above that eigenvalues of $\left(\widehat{\mathbf{U}^{*}(t)}\right)^{*}: H^{+}(\mathbb{R}) \mapsto H^{+}(\mathbb{R})$, are generalized eigenvalues of $\mathbf{U}(t)$ (for $t \geq 0)$.

2. Obviously, the 1.h.s. of Eq. (2.5) can be defined for every $t \in(-\infty, \infty)$. Eq. (2.5), however, is valid for $t \geq 0$.

Proof of Theorem 2.1: 
At the center of the proof of Theorem 2.1 lie two theorems, proved by C. Van Winter, that enable us to define a contractive nesting of $L^{2}\left(\mathbb{R}^{+}\right)$into $H^{+}(\mathbb{R})$. Taken together, the following theorems are referred to below as the Van Winter Theorem [VW]:

Theorem 2.2 (C. Van Winter) The class of functions $f$ which are analytic, regular in the upper half-plane, and have the property that

$$
\int_{0}^{\infty} d r\left|f\left(r e^{i \phi}\right)\right|^{2}
$$

is uniformly bounded in $\phi$ for $0<\phi<\pi$, is identical to the Hardy class $H^{2}(\Pi)$. For each such $f$ there exists a boundary value function $f(r)(r>0)$ on $\mathbb{R}^{+}$. The boundary values $f(r)$ of functions $f\left(r e^{i \phi}\right)$ in $H^{2}(\Pi)$ are dense in $L^{2}\left(\mathbb{R}^{+}\right)$.

Theorem 2.3 (C. Van Winter) A complex valued function $f$ defined on the upper half-plane $\Pi$ is in $H^{2}(\Pi)$ if and only if it is given by an inverse Mellin transform as

$$
f\left(r e^{i \phi}\right)=\frac{1}{\sqrt{2 \pi}} \int_{-\infty}^{+\infty} d s E(s)\left(r e^{i \phi}\right)^{-i s-\frac{1}{2}}
$$

for some function $E(s)$ satisfying

$$
\int_{-\infty}^{+\infty} d s\left(1+e^{2 \pi s}\right)|E(s)|^{2}<\infty
$$

in this case the Mellin transform of $f$ is given by

$$
E(s, \phi)=\frac{1}{\sqrt{2 \pi}} \int_{0}^{\infty} d r f\left(r e^{i \phi}\right) r^{i s-\frac{1}{2}}=e^{\phi s-i \frac{\phi}{2}} E(s)
$$

It can be shown [VW] that in Eq. (2.9) it is possible to take $\phi$ to zero, i.e., that if $f(r)$ is the boundary value on $\mathbb{R}^{+}$of a function $f\left(r e^{i \phi}\right)$ in $H^{2}(\Pi)$ then

$$
E(s)=\frac{1}{\sqrt{2 \pi}} \int_{0}^{\infty} d r f(r) r^{i s-\frac{1}{2}}
$$

By combining Theorem 2.2 and Theorem 2.3 we find that any function in $H^{2}(\Pi)$ can be reconstructed from its boundary value on the positive real axis. Define $\theta: H^{+}(\mathbb{R}) \mapsto L^{2}\left(\mathbb{R}^{+}\right)$by taking, for each function $f \in H^{2}(\Pi)$, the restriction to $\mathbb{R}^{+}$of the boundary value of $f$ on the real axis. Then $\theta$ is one to one and $\theta^{-1}$ can be defined. It is also important to note that the Van Winter theorem can be applied to boundary values of Hardy class functions on the negative real axis, a fact which is used below.

Using the Van Winter Theorem we easily obtain the following result: 
Proposition 2.1 The map $\theta: H^{+}(\mathbb{R}) \rightarrow L^{2}\left(\mathbb{R}^{+}\right)$is a contractive nesting map, $\left(H^{+}(\mathbb{R}), L^{2}\left(\mathbb{R}^{+}\right), \theta\right)$ is a contractive nesting of $H^{+}(\mathbb{R})$ into $L^{2}\left(\mathbb{R}^{+}\right)$ and $\left(L^{2}\left(\mathbb{R}^{+}\right), H^{+}(\mathbb{R}), \theta^{*}\right)$ is a contractive nesting of $L^{2}\left(\mathbb{R}^{+}\right)$into $H^{+}(\mathbb{R})$.

Proof:

From the Van Winter Theorem we know that the map $\theta$ is injective and that $\theta H^{+}(\mathbb{R}) \subset L^{2}\left(\mathbb{R}^{+}\right)$is dense in $L^{2}\left(\mathbb{R}^{+}\right)$. Denote by $H^{-}(\mathbb{R})$ the space of boundary values on $\mathbb{R}$ of functions belonging to $H^{2}(\bar{\Pi})$ (recall that $\bar{\Pi}$ is the lower half of the complex plane). We have

$$
L^{2}(\mathbb{R})=H^{-}(\mathbb{R}) \oplus H^{+}(\mathbb{R})
$$

$H^{+}(\mathbb{R})$ is then a subapce of $L^{2}(\mathbb{R})$ and it inherits its scalar product from that of $L^{2}(\mathbb{R})$. By the definition of $\theta$ we have for $f \in H^{+}(\mathbb{R})$

$$
\|\theta f\|_{L^{2}\left(\mathbb{R}^{+}\right)}^{2}=(\theta f, \theta f)_{L^{2}\left(\mathbb{R}^{+}\right)}<(f, f)_{L^{2}(\mathbb{R})}=(f, f)_{H^{+}(\mathbb{R})}=\|f\|_{H^{+}(\mathbb{R})}^{2} .
$$

$\theta: H^{+}(\mathbb{R}) \mapsto L^{2}\left(\mathbb{R}^{+}\right)$is therefore a contraction and $\left(H^{+}(\mathbb{R}), L^{2}\left(\mathbb{R}^{+}\right), \theta\right)$ is a contractive nesting of $H^{+}(\mathbb{R})$ into $L^{2}\left(\mathbb{R}^{+}\right)$. Defining the adjoint map $\theta^{*}: L^{2}\left(\mathbb{R}^{+}\right) \mapsto H^{+}(\mathbb{R})$ through the relation

$$
(\theta x, y)_{L^{2}\left(\mathbb{R}^{+}\right)}=\left(x, \theta^{*} y\right)_{H^{+}(\mathbb{R})} \quad x \in H^{+}(\mathbb{R}), y \in L^{2}\left(\mathbb{R}^{+}\right)
$$

we conclude that $\left(L^{2}\left(\mathbb{R}^{+}\right), H^{+}(\mathbb{R}), \theta^{*}\right)$ is a contractive nesting of $L^{2}\left(\mathbb{R}^{+}\right)$ into $H^{+}(\mathbb{R})$.

Consider the spectral (or energy) representation for $\mathbf{H}_{0}$. The assumption $\sigma_{a c}\left(\mathbf{H}_{0}\right)=\mathbb{R}^{+}$(and that the multiplicity is one) leads to the existence of a unitary map $U: \mathcal{H}_{a c}^{0} \mapsto L^{2}\left(\mathbb{R}^{+}\right)$defining a representation of $\mathcal{H}_{a c}^{0}$ in terms of the space $L^{2}\left(\mathbb{R}^{+}\right)$. We can now use the unitarity of the Møller wave operator $\boldsymbol{\Omega}^{+}: \mathcal{H}_{a c}^{0} \mapsto \mathcal{H}_{a c}$ and define a unitary map $\mathbf{W}^{+}: \mathcal{H}_{a c} \mapsto L^{2}\left(\mathbb{R}^{+}\right)$by

$$
\mathbf{W}^{+} \equiv U\left(\boldsymbol{\Omega}^{+}\right)^{*}
$$

$\mathbf{W}^{+}$is a transformation to a spectral representation for $\mathbf{H} \mid \mathcal{H}_{a c}$. Applying the contractive nesting $\left(L^{2}\left(\mathbb{R}^{+}\right), H^{+}(\mathbb{R}), \theta^{*}\right)$ then allows us to define a map

$$
\hat{\mathbf{\Omega}}_{+}: \mathcal{H}_{a c} \mapsto H^{+}(\mathbb{R})
$$

by

$$
\hat{\boldsymbol{\Omega}}_{+} \equiv \theta^{*} \mathbf{W}^{+}=\theta^{*} U\left(\boldsymbol{\Omega}^{+}\right)^{*}
$$


Since $\mathbf{W}^{+}$is unitary and $\theta^{*}$ is a contractive nesting map we find that $\left(\mathcal{H}_{a c}, H^{+}(\mathbb{R}), \hat{\mathbf{\Omega}}_{+}\right)$is a contractive nesting of $\mathcal{H}_{a c}$ into $H^{+}(\mathbb{R})$ and $(\alpha)$ is proved.

Next, we turn to the proof of $(\beta)$. Assume that the range of $\hat{\Omega}_{+}^{*}$ is invariant for an operator $\mathbf{A}: \mathcal{H}_{a c} \mapsto \mathcal{H}_{a c}$. Then, it is possible to use the nesting $\left(H^{+}(\mathbb{R}), \mathcal{H}_{a c}, \hat{\mathbf{\Omega}}_{+}^{*}\right)$ to define the Grossmann type operator $\hat{\mathbf{A}}: H^{+}(\mathbb{R}) \mapsto H^{+}(\mathbb{R})$ according to Eq. (2.3), i.e.,

$$
\hat{\mathbf{A}}=\left(\hat{\mathbf{\Omega}}_{+}^{*}\right)^{-1} \mathbf{A} \hat{\mathbf{\Omega}}_{+}^{*} .
$$

The generalized eigenvalues of $\mathbf{A}$ are then eigenvalues of the operator ${\widehat{\left(\mathbf{A}^{*}\right)}}^{*}$ : $H^{+}(\mathbb{R}) \mapsto H^{+}(\mathbb{R})$ given by

$$
{\widehat{\left(\mathbf{A}^{*}\right)}}^{*}=\hat{\mathbf{\Omega}}_{+} \mathbf{A}^{* *}\left(\left(\hat{\mathbf{\Omega}}_{+}^{*}\right)^{-1}\right)^{*} .
$$

The first equality in Eq. (2.5) results from the following proposition

Proposition 2.2 For any bounded operator A : $\mathcal{H}_{a c} \mapsto \mathcal{H}_{a c}$ satisfying $\mathbf{A}^{* *}=\mathbf{A}$ and such that $\hat{\mathbf{\Omega}}_{+}^{*} H^{+}(\mathbb{R}) \subset \mathcal{H}_{a c}$ is invariant for $\mathbf{A}$, and for any $h \in \mathcal{H}_{a c}$ we have

$$
\left(\widehat{\mathbf{A}^{*}}\right)^{*} \hat{\mathbf{\Omega}}_{+} h=\hat{\mathbf{\Omega}}_{+} \mathbf{A} h .
$$

Proof:

Since $\hat{\boldsymbol{\Omega}}_{+}^{*}$ is a nesting map of $H^{+}(\mathbb{R})$ into $\mathcal{H}_{a c}$ the map $\left(\hat{\boldsymbol{\Omega}}_{+}^{*}\right)^{-1}$ is defined on the dense set $\hat{\boldsymbol{\Omega}}_{+}^{*} H^{+}(\mathbb{R}) \subset \mathcal{H}_{a c}$ and we can define the map $\left(\left(\hat{\boldsymbol{\Omega}}_{+}^{*}\right)^{-1}\right)^{*}$ through the relation

$$
(g, f)_{H^{+}(\mathbb{R})}=\left(\left(\hat{\boldsymbol{\Omega}}_{+}^{*}\right)^{-1} \hat{\boldsymbol{\Omega}}_{+}^{*} g, f\right)_{H^{+}(\mathbb{R})}=\left(\hat{\mathbf{\Omega}}_{+}^{*} g,\left(\left(\hat{\mathbf{\Omega}}_{+}^{*}\right)^{-1}\right)^{*} f\right)_{\mathcal{H}_{a c}}
$$

which holds for any $f \in D\left(\left(\left(\hat{\boldsymbol{\Omega}}_{+}^{*}\right)^{-1}\right)^{*}\right)$ and any $g \in H^{+}(\mathbb{R})$. It is then true that

$$
\left(\left(\hat{\mathbf{\Omega}}_{+}^{*}\right)^{-1}\right)^{*}=\left(\hat{\mathbf{\Omega}}_{+}^{* *}\right)^{-1}=\hat{\mathbf{\Omega}}_{+}^{-1} .
$$

From Eq. (2.16) and Eq. (2.17) we conclude that, for any $f \in \hat{\boldsymbol{\Omega}}_{+} \mathcal{H}_{a c}$ we have

$$
{\widehat{\left(\mathbf{A}^{*}\right)}}^{*} f=\hat{\mathbf{\Omega}}_{+} \mathbf{A}^{* *} \hat{\mathbf{\Omega}}_{+}^{-1} f \quad f \in \hat{\mathbf{\Omega}}_{+} \mathcal{H}_{a c} .
$$

By the injective property of $\hat{\boldsymbol{\Omega}}_{+}$, for any $f \in \hat{\boldsymbol{\Omega}}_{+} \mathcal{H}_{a c}$ there is a unique $h \in \mathcal{H}_{a c}$ such that $f=\hat{\boldsymbol{\Omega}}_{+} h$. Hence, by Eq. (2.18) we have

$$
{\widehat{\left(\mathbf{A}^{*}\right)}}^{*} \hat{\mathbf{\Omega}}_{+} h=\hat{\mathbf{\Omega}}_{+} \mathbf{A}^{* *} h \quad h \in \mathcal{H}_{a c} .
$$


In particular, if $\mathbf{A}$ satisfies $\mathbf{A}^{* *}=\mathbf{A}$ we find that

$$
\widehat{(\mathbf{A}}^{*} \hat{\mathbf{\Omega}}_{+} h=\hat{\mathbf{\Omega}}_{+} \mathbf{A} h \quad h \in \mathcal{H}_{a c} .
$$

It is easy to see that, for $t \geq 0, \mathbf{U}(t) \hat{\boldsymbol{\Omega}}_{+}^{*} H^{+}(\mathbb{R}) \subset \hat{\mathbf{\Omega}}_{+}^{*} H^{+}(\mathbb{R})$. Indeed we have

$$
\begin{aligned}
\mathbf{U}(t) \hat{\mathbf{\Omega}}_{+}^{*} H^{+}(\mathbb{R}) & =\mathbf{U}(t)\left(\mathbf{W}^{+}\right)^{*} \theta H^{+}(\mathbb{R})= \\
& =\left(\mathbf{W}^{+}\right)^{*} \bar{u}(t) \theta H^{+}(\mathbb{R}) \subset\left(\mathbf{W}^{+}\right)^{*} \theta H^{+}(\mathbb{R})=\hat{\mathbf{\Omega}}_{+}^{*} H^{+}(\mathbb{R})
\end{aligned}
$$

hence, we can apply Proposition 2.2 to $\mathbf{U}(t)(t \geq 0)$ and obtain the first equality in Eq. (2.5).

In order to prove the second equality in Eq. (2.5) we need to obtain a more explicit expression for the map $\hat{\boldsymbol{\Omega}}_{+}$. We have

Proposition 2.3 Define the inclusion map $\mathbf{I}: L^{2}\left(\mathbb{R}^{+}\right) \mapsto L^{2}(\mathbb{R})$ by

$$
(\mathbf{I} \phi)(\sigma)=\left\{\begin{array}{ll}
\phi(\sigma) & \sigma \geq 0, \\
0 & \sigma<0 .
\end{array} \quad, \phi \in L^{2}\left(\mathbb{R}^{+}\right) .\right.
$$

Let $\mathbf{P}_{+}$be the orthogonal projection of $L^{2}(\mathbb{R})$ into $H^{+}(\mathbb{R})$ and let $\mathbf{W}^{+}$be the unitary map defined in Eq. (2.13). Then the map $\hat{\mathbf{\Omega}}_{+}: \mathcal{H}_{a c} \mapsto H^{+}(\mathbb{R})$ is given by

$$
\hat{\mathbf{\Omega}}_{+}=\mathbf{P}_{+} \mathbf{I} \mathbf{W}^{+} \text {. }
$$

\section{Proof:}

We first need an explicit expression for the map $\theta^{*}$. Regarding $L^{2}\left(\mathbb{R}^{+}\right)$ as a closed subspace of $L^{2}(\mathbb{R})$, the inclusion map $\mathbf{I}: L^{2}\left(\mathbb{R}^{+}\right) \mapsto L^{2}(\mathbb{R})$ is given by Eq. (2.21). The l.h.s. of Eq. (2.12) can be written then

$$
(\theta x, y)_{L^{2}\left(\mathbb{R}^{+}\right)}=(\mathbf{I} \theta x, \mathbf{I} y)_{L^{2}(\mathbb{R})} .
$$

Making use of the decomposition of $L^{2}(\mathbb{R})$, Eq. (2.10), we observe that any $y \in L^{2}\left(\mathbb{R}^{+}\right)$correspond to the following decompositon into an orthogonal sum

$$
\mathbf{I} y=y_{+}+y_{-} \quad y_{+} \in H^{+}(\mathbb{R}), y_{-} \in H^{-}(\mathbb{R})
$$

Eq. (2.21) then implies that

$$
y_{+}(\sigma)=-y_{-}(\sigma) \quad \sigma<0 .
$$


Inserting the decomposition, Eq. (2.24), into the r.h.s. of Eq. (2.23) we obtain

$$
(\theta x, y)_{L^{2}\left(\mathbb{R}^{+}\right)}=\left(\mathbf{I} \theta x, y_{+}+y_{-}\right)_{L^{2}(\mathbb{R})}=\int_{-\infty}^{+\infty} d \sigma\left((\mathbf{I} \theta x)(\sigma), y_{+}(\sigma)+y_{-}(\sigma)\right)
$$

We can now use Eq. (2.26) to obtain the following identity

$$
\begin{array}{r}
\int_{-\infty}^{+\infty} d \sigma\left((\mathbf{I} \theta x)(\sigma), y_{+}(\sigma)+y_{-}(\sigma)\right)=\int_{-\infty}^{+\infty} d \sigma\left(x(\sigma), y_{+}(\sigma)+y_{-}(\sigma)\right)= \\
=\int_{-\infty}^{+\infty} d \sigma\left(x(\sigma), y_{+}(\sigma)\right)=\left(x, y_{+}\right)_{H^{+}(\mathbb{R})}
\end{array}
$$

where the second equality follows from the orthogonality of $H^{+}(\mathbb{R})$ and $H^{-}(\mathbb{R})$. Eq. (2.26)-(2.27) imply that

$$
(\theta x, y)_{L^{2}\left(\mathbb{R}^{+}\right)}=\left(x, y_{+}\right)_{H^{+}(\mathbb{R})} .
$$

Comparing Eq. (2.12) and Eq. (2.28) we find the desired expression for the $\operatorname{map} \theta^{*}: L^{2}\left(\mathbb{R}^{+}\right) \mapsto H^{+}(\mathbb{R})$

$$
\theta^{*} y=y_{+}=\mathbf{P}_{+} \mathbf{I} y \quad, y \in L^{2}\left(\mathbb{R}^{+}\right), y_{+} \in H^{+}(\mathbb{R}) .
$$

Eq. (2.14) can now be written in the form

$$
\hat{\Omega}_{+}=\mathbf{P}_{+} \mathbf{I} \mathbf{W}^{+} .
$$

Observe that, for any $h \in \mathcal{H}_{a c}$ we have

$$
\mathbf{I W}^{+} \mathbf{U}(t) h=\mathbf{I} U \boldsymbol{\Omega}_{+}^{*} \mathbf{U}(t) h=\mathbf{I} U \mathbf{U}_{\mathbf{0}}(t) \boldsymbol{\Omega}_{+}^{*} h=u(t) \mathbf{I} \mathbf{W}^{+} h
$$

where $u(t)$ is the multiplicative operator defined in Eq. (1.14). Using again the decomposition in Eq. (2.10) we have

$$
\mathbf{I W}^{+} h=\mathbf{P}_{+} \mathbf{I W}^{+} h+\mathbf{P}_{-} \mathbf{I} \mathbf{W}^{+} h=h_{+}+h_{-}
$$

where $h_{+}=\mathbf{P}_{+} \mathbf{I W}^{+} h=\hat{\mathbf{\Omega}}^{+} h$ and $h_{-}=\mathbf{P}_{-} \mathbf{I W}^{+} h$. Here $\mathbf{P}_{+}$is the projection on $H^{+}(\mathbb{R})$ defined above and $\mathbf{P}_{-}: L^{2}(\mathbb{R}) \mapsto H^{-}(\mathbb{R})$ is the orthogonal projection of $L^{2}(\mathbb{R})$ on $H^{-}(\mathbb{R})$. From Eq. (2.30)-(2.32) we obtain, for any $h \in \mathcal{H}_{a c}$,

$$
\hat{\mathbf{\Omega}}_{+} \mathbf{U}(t) h=\mathbf{P}_{+} u(t) \mathbf{I} \mathbf{W}^{+} h=\mathbf{P}_{+} u(t)\left(h_{+}+h_{-}\right) .
$$


We conclude the proof of Theorem 2.1 by observing that $H^{-}(\mathbb{R})$ is stable under the action of $u(t)$ for $t \geq 0$. Hence we have $u(t) h_{-} \in H^{-}(\mathbb{R})$ and so

$$
\hat{\mathbf{\Omega}}_{+} \mathbf{U}(t) h=\mathbf{P}_{+} u(t) h_{+}=T_{u(t)} \hat{\mathbf{\Omega}}_{+} h .
$$

This completes the proof of Theorem 2.1.

Theorem 2.1 provides the analogue of the Fourier transform of the representation in Theorem 1.1 in Section 1, that is, it is the analogue of the outgoing spectral representation of the Lax-Phillips scattering theory. One may think of it as the "outgoing contractive nesting" associated with $\mathcal{H}_{a c}$ and $\mathbf{U}(t)$.

As in the case of the Lax-Phillips theory, there is another nesting structure which may be thought of as the "incoming contractive nesting" associated with $\mathcal{H}_{a c}$ and $\mathbf{U}(t)$. In a similar fashion to Eq. (2.14) we define a contractive nesting map $\hat{\boldsymbol{\Omega}}_{-}: \mathcal{H}_{a c} \mapsto H^{+}(\mathbb{R})$ by

$$
\hat{\mathbf{\Omega}}_{-} \equiv \theta^{*} \mathbf{W}^{-}=\theta^{*} U\left(\boldsymbol{\Omega}^{-}\right)^{*} .
$$

For $\hat{\boldsymbol{\Omega}}_{-}$we have the following theorem:

Theorem 2.4 (incoming contractive nesting) Let $\mathbf{H}_{0}$ and $\mathbf{H}$ be selfadjoint operators on a Hilbert space $\mathcal{H}$ and let $\{\mathbf{U}(t)\}_{t \in \mathbb{R}}$ be the unitary evolution group on $\mathcal{H}$ generated by $\mathbf{H}$. Denote by $\mathcal{H}_{a c}^{0}$ and $\mathcal{H}_{\text {ac }}$, respectively. the absolutely continuous subspaces of $\mathbf{H}_{0}$ and $\mathbf{H}$. Assume that the absolutely continuous spectrum of $\mathbf{H}_{0}$ and $\mathbf{H}$ has multiplicity one and that it satisfies $\sigma_{a c}\left(\mathbf{H}_{0}\right)=\sigma_{a c}(\mathbf{H})=\mathbb{R}^{+}$. Assume furthermore that the Møller wave operator $\boldsymbol{\Omega}^{+} \equiv \boldsymbol{\Omega}^{+}\left(\mathbf{H}_{0}, \mathbf{H}\right): \mathcal{H}_{a c}^{0} \mapsto \mathcal{H}_{a c}$ exists and is complete. Then there exists a map $\hat{\boldsymbol{\Omega}}_{-}: \mathcal{H}_{a c} \mapsto H^{+}(\mathbb{R})$ such that

$(\alpha)\left(\mathcal{H}_{a c}, H^{+}(\mathbb{R}), \hat{\mathbf{\Omega}}_{-}\right)$is a contractive Hilbert space nesting of $\mathcal{H}_{a c}$ into $H^{+}(\mathbb{R})$.

( $\beta$ ) For every $t \geq 0$ define the Grossmann type operator $\widehat{\mathbf{U}^{*}}(t): H^{+}(\mathbb{R}) \mapsto$ $H^{+}(\mathbb{R})$ according to Eq. (2.3), i.e., $\widehat{\mathbf{U}^{*}}(t)=\left(\hat{\mathbf{\Omega}}_{-}^{*}\right)^{-1} \mathbf{U}^{*}(t) \hat{\mathbf{\Omega}}_{-}^{*}$. These operators are well defined and, for every $t \geq 0$ and every $f \in \mathcal{H}_{\text {ac }}$ we have

$$
\hat{\boldsymbol{\Omega}}_{-} \mathbf{U}(t) f=\left(\widehat{\mathbf{U}^{*}(t)}\right)^{*} \hat{\boldsymbol{\Omega}}_{-} f=T_{u(t)} f_{i n} \quad t \geq 0
$$

where $f_{\text {in }}=\hat{\mathbf{\Omega}}_{-} f$ and $T_{u(t)}$ is the Toeplitz operator with symbol $u(t)$ given by Eq. (1.14). 
Once the existence of the incoming contractive nesting and the outgoing contractive nesting are established we can turn to the definition of the analogue of the Lax-Phillips $S$-matrix. Let $f_{\text {in }}=\hat{\boldsymbol{\Omega}}_{-} f \in H^{+}(\mathbb{R})$ be the "incoming nested representer" of an element $f \in \mathcal{H}_{a c}$. Let $f_{\text {out }}=\hat{\boldsymbol{\Omega}}_{+} f \in H^{+}(\mathbb{R})$ be the "outgoing nested representer" of the same element $f$. We have

$$
\begin{aligned}
f_{\text {out }}=\hat{\mathbf{\Omega}}_{+} \hat{\boldsymbol{\Omega}}_{-}^{-1} f_{\text {in }}=\theta^{*} U\left(\boldsymbol{\Omega}^{-}\right)^{*} \boldsymbol{\Omega}^{+} U^{*}\left(\theta^{*}\right)^{-1} f_{\text {in }}= & \\
& =\theta^{*} U \mathbf{S} U^{*}\left(\theta^{*}\right)^{-1} f_{\text {in }}=\theta^{*} \tilde{\mathbf{S}}\left(\theta^{*}\right)^{-1} f_{\text {in }}
\end{aligned}
$$

where $\mathbf{S}=\left(\boldsymbol{\Omega}^{-}\right)^{*} \boldsymbol{\Omega}^{+}$is the scattering operator and $\tilde{\mathbf{S}}: L^{2}\left(\mathbb{R}^{+}\right) \mapsto L^{2}\left(\mathbb{R}^{+}\right)$ is defined by $\tilde{\mathbf{S}} \equiv U \mathbf{S} U^{*}$. The operator $\tilde{\mathbf{S}}$ is the representer of $\mathbf{S}$ in the spectral (energy) representation for $\mathbf{H}_{0}$ and is a multiplicative operator in this representation, i.e.,

$$
(\tilde{\mathbf{S}} f)(E)=\tilde{\mathbf{S}}(E) f(E) \quad, f \in L^{2}\left(\mathbb{R}^{+}\right) .
$$

Eq. (2.37) provides the definition of the nested scattering matrix, i.e.,

Definition (nested scattering matrix): Let $\mathbf{S}=\left(\boldsymbol{\Omega}^{-}\right)^{*} \boldsymbol{\Omega}^{+}$be the scattering operator associated with $\mathbf{H}_{0}$ and $\mathbf{H}$. Let $\tilde{\mathbf{S}}: L^{2}\left(\mathbb{R}^{+}\right) \mapsto L^{2}\left(\mathbb{R}^{+}\right)$be given by

$$
\tilde{\mathbf{S}} \equiv U \mathbf{S} U^{*}
$$

The nested scattering matrix $\mathbf{S}_{\text {nest }}: H^{+}(\mathbb{R}) \mapsto H^{+}(\mathbb{R})$ is defined to be

$$
\mathbf{S}_{n e s t} \equiv \theta^{*} \tilde{\mathbf{S}}\left(\theta^{*}\right)^{-1} \text {. }
$$

Of course, for every $f \in \mathcal{H}_{a c}$ we have $f_{\text {out }}=\mathbf{S}_{\text {nest }} f_{\text {in }}$ where $f_{\text {out }}=\hat{\mathbf{\Omega}}_{+} f$ and $f_{\text {in }}=\hat{\mathbf{\Omega}}_{-} f$. We have the following theorem:

Theorem 2.5 Consider $L^{2}\left(\mathbb{R}^{+}\right)$and $L^{2}\left(\mathbb{R}^{-}\right)$as closed subspaces of $L^{2}(\mathbb{R})$. Denote $\mathbf{P}_{\mathbb{R}^{+}}: L^{2}(\mathbb{R}) \mapsto L^{2}(\mathbb{R})$ the orthogonal projection of $L^{2}(\mathbb{R})$ on $L^{2}\left(\mathbb{R}^{+}\right)$ and, similarly, $\mathbf{P}_{\mathbb{R}^{-}}: L^{2}(\mathbb{R}) \mapsto L^{2}(\mathbb{R})$ the orthogonal projection of $L^{2}(\mathbb{R})$ on $L^{2}\left(\mathbb{R}^{-}\right)$. Let $\tilde{\mathbf{S}}$ be defined as in Eq. (2.38). Then, for every $f_{\text {in }} \in \hat{\mathbf{\Omega}}_{-} \mathcal{H}_{a c} \subset$ $H^{+}(\mathbb{R})$ we have

$$
\begin{aligned}
& {\left[\mathbf{S}_{n e s t} f_{i n}\right](\sigma)=\frac{1}{2 \pi} \int_{0}^{\infty} d E \frac{i}{\sigma-E+i 0^{+}} \tilde{\mathbf{S}}(E)\left[\mathbf{P}_{\mathbb{R}^{+}} f_{i n}\right](\sigma)+\frac{1}{4 \pi^{2}} \times} \\
& \int_{0}^{\infty} d E \frac{i}{\sigma-E+i 0^{+}} \tilde{\mathbf{S}}(E)\left\{\int_{-\infty}^{\infty} d s \int_{-\infty}^{0} d \sigma^{\prime}\left[\mathbf{P}_{\mathbb{R}^{-}} f_{i n}\right]\left(\sigma^{\prime}\right) \sigma^{-i s-\frac{1}{2}} E^{i s-\frac{1}{2}}\right\} .
\end{aligned}
$$




\section{Proof:}

we need to obtain a realization of the maps $\theta^{*}$ and $\left(\theta^{*}\right)^{-1}$ on the functional level. For $\theta^{*}$ we have

Lemma 2.1 The map $\theta^{*}: L^{2}\left(\mathbb{R}^{+}\right) \mapsto H^{+}(\mathbb{R})$ is given, for any $f \in L^{2}\left(\mathbb{R}^{+}\right)$, by

$$
\left(\theta^{*} f\right)(\sigma)=\frac{1}{2 \pi} \int_{0}^{\infty} d E \frac{i}{\sigma-E+i 0^{+}} f(E)
$$

Proof: The starting point is Eq. (2.29). The inclusion map $\mathbf{I}: L^{2}\left(\mathbb{R}^{+}\right) \mapsto$ $L^{2}(\mathbb{R})$ is realized in a simple way: given a function $f \in L_{\mathcal{N}}^{2}\left(\mathbb{R}^{+}\right)$, and for any $s \in \mathbb{R}$, we have

$$
(\mathbf{I} f)(s)=\int_{0}^{\infty} d E \delta(s-E) f(E)
$$

where $\delta(\cdot)$ is the Dirac delta distribution.

Now for the realization of the projection $\mathbf{P}_{+}: L^{2}(\mathbb{R}) \mapsto H^{+}(\mathbb{R})$. For this we Fourier transform $L^{2}(\mathbb{R})$ and note that, denoting the Fourier transform operator by $\mathbf{F}$, we have $\mathbf{F} H^{+}(7)=L^{2}\left(\mathbb{R}^{+}\right)$and $\mathbf{F} H^{-}(\mathbb{R})=L^{2}\left(\mathbb{R}^{-}\right)$. For any $f \in L^{2}(\mathbb{R})$ we therefore obtain

$$
\mathbf{P}_{+} f=\mathbf{F}^{-1} \mathbf{P}_{\mathbb{R}^{+}} \mathbf{F} f
$$

and hence, for $f \in L^{2}(\mathbb{R})$

$$
\begin{aligned}
& \left(\mathbf{P}_{+} f\right)(\sigma)=\frac{1}{2 \pi} \int_{-\infty}^{\infty} d t e^{i \sigma t}\left(\theta(t) \int_{-\infty}^{\infty} d s e^{-i t s} f(s)\right)= \\
& \quad=\frac{1}{2 \pi} \int_{-\infty}^{\infty} d s \int_{0}^{\infty} d t e^{i\left(\sigma-s+i 0^{+}\right) t} f(s)=\frac{1}{2 \pi} \int_{-\infty}^{\infty} d s \frac{i}{\sigma-s+i 0^{+}} f(s) .
\end{aligned}
$$

Combining Eq. (2.41) and Eq. (2.42) we get, for $f \in L^{2}\left(\mathbb{R}^{+}\right)$

$$
\begin{aligned}
&\left(\theta^{*} f\right)(\sigma)=\left(\mathbf{P}_{+} \mathbf{I} f\right)(\sigma)= \\
&=\frac{1}{2 \pi} \int_{-\infty}^{\infty} d s \frac{i}{\sigma-s+i 0^{+}}\left[\int_{0}^{\infty} d E \delta(s-E) f(E)\right]= \\
&=\frac{1}{2 \pi} \int_{0}^{\infty} d E \frac{i}{\sigma-E+i 0^{+}} f(E)
\end{aligned}
$$

Next, we need to find an explicit expression for the inverse map $\left(\theta^{*}\right)^{-1}$. We have the following lemma: 
Lemma 2.2 The map $\left(\theta^{*}\right)^{-1}: \theta^{*} L^{2}\left(\mathbb{R}^{+}\right) \mapsto L^{2}\left(\mathbb{R}^{+}\right)$is given, for any $f \in$ $\theta^{*} L^{2}\left(\mathbb{R}^{+}\right)$by

$$
\left[\left(\theta^{*}\right)^{-1} f\right](E)=\left[\mathbf{P}_{\mathbb{R}^{+}} f\right](E)+\frac{1}{2 \pi} \int_{-\infty}^{\infty} d s \int_{-\infty}^{0} d \sigma\left[\mathbf{P}_{\mathbb{R}^{-}} f\right](\sigma) \sigma^{-i s-\frac{1}{2}} E^{i s-\frac{1}{2}}
$$

where $E \geq 0, \mathbf{P}_{\mathbb{R}^{+}}$and $\mathbf{P}_{\mathbb{R}^{-}}$are the orthogonal projections of $L^{2}(\mathbb{R})$ on $L^{2}\left(\mathbb{R}^{+}\right)$and $L^{2}\left(\mathbb{R}^{-}\right)$respectively $\left(L^{2}\left(\mathbb{R}^{+}\right)\right.$and $L^{2}\left(\mathbb{R}^{-}\right)$are considered as subspaces of $L^{2}(\mathbb{R})$.)

Proof: The procedure for constructing $\left(\theta^{*}\right)^{-1}$ is as follows: Given $y_{+} \in$ $H^{+}(\mathbb{R})$ in the range of $\theta^{*}$, there is some vector $y \in L^{2}\left(\mathbb{R}^{+}\right)$such that $\mathbf{I} y=$ $y_{+}+y_{-}\left(y_{-}\right.$is in $\left.H^{-}(\mathbb{R})\right)$ and Eq. (2.25) is valid. $y_{-}(\sigma)$ for $\sigma<0$ are then the restriction to $\mathbb{R}^{-}$of the values of some function $y_{-} \in H^{-}(\mathbb{R})$. Van Winter's theorem then implies that $y_{-} \in H^{2}(\bar{\Pi})$, and hence also $y_{-} \in H^{-}(\mathbb{R})$, can be uniquely reconstructed from its boundary value on the negative real axis. We conclude that, for each $y_{+}$in the range of $\theta^{*}$ we can assign a unique $y_{-} \in H^{-}(\mathbb{R})$ such that Eq. (2.25) holds. Therefore, $y=y_{+}+y_{-}$can be identified with a unique element in $L^{2}\left(\mathbb{R}^{+}\right)$corresponding to a given $y_{+} \in \theta^{*} L^{2}\left(\mathbb{R}^{+}\right)$. This construction defines an inverse $\left(\theta^{*}\right)^{-1}$ such that

$$
\left(\theta^{*}\right)^{-1} y_{+}=y \quad, y_{+} \in \theta^{*} L^{2}\left(\mathbb{R}^{+}\right), y \in L^{2}\left(\mathbb{R}^{+}\right) .
$$

Observe that the main step in the procedure described above is the construction, through the Van Winter Theorem, of a function $f \in H^{2}(\bar{\Pi})$ from data on the boundary value function of $f$ on the negative real axis $\mathbb{R}^{-}$.

Given the boundary value function $\theta h \in L^{2}\left(\mathbb{R}^{+}\right)$for a function $h \in$ $H^{2}(\Pi)$, Van Winter's theorem implies that one can reconstruct the function $h \in H^{2}(\Pi)$ by using an inverse Mellin transform. We first find the Mellin transform

$$
E(s)=\frac{1}{\sqrt{2 \pi}} \int_{0}^{\infty} h(x) x^{i s-\frac{1}{2}} d x
$$

and then we reconstruct the analytic function via the inverse Mellin transform as follows

$$
h\left(r e^{i \phi}\right)=\frac{1}{\sqrt{2 \pi}} \int_{-\infty}^{\infty} E(s)\left(r e^{i \phi}\right)^{-i s-\frac{1}{2}} d s \quad z=r e^{i \phi}, r>0,0 \leq \phi \leq \pi
$$

For a function $h \in H^{2}(\Pi)$, define a new function by $\tilde{h}(z)=\bar{h}(\bar{z}), \operatorname{Im} z<0$. Then $\tilde{h} \in H^{2}(\bar{\Pi})$. In fact, each function $\tilde{h} \in H^{2}(\bar{\Pi})$ can be obtained from 
some function $h \in H^{2}(\Pi)$ by this procedure. From Eq. (2.44) and Eq. (2.45) we obtain

$$
\bar{E}(s)=\frac{1}{\sqrt{2 \pi}} \int_{0}^{\infty} \bar{h}(x) x^{-i s-\frac{1}{2}} d x
$$

and

$$
\bar{h}\left(\overline{r e^{i \phi}}\right)=\frac{1}{\sqrt{2 \pi}} \int_{-\infty}^{\infty} \bar{E}(s)\left(r e^{i \phi}\right)^{i s-\frac{1}{2}} d s \quad z=r e^{i \phi}, r \geq 0,-\pi \leq \phi \leq 0 .
$$

Denoting $\tilde{E}(s)=\bar{E}(s)$ and taking notice of the fact that $\tilde{h}(x)=\bar{h}(x)$ for real $x$, we can write Eq. (2.46) and Eq. (2.47) in the form

$$
\tilde{E}(s)=\frac{1}{\sqrt{2 \pi}} \int_{0}^{\infty} \tilde{h}(x) x^{-i s-\frac{1}{2}} d x
$$

and

$$
\tilde{h}\left(r e^{i \phi}\right)=\frac{1}{\sqrt{2 \pi}} \int_{-\infty}^{\infty} \tilde{E}(s)\left(r e^{i \phi}\right)^{i s-\frac{1}{2}} d s \quad z=r e^{i \phi}, r \geq 0,-\pi \leq \phi \leq 0 .
$$

Using Eq. (2.48) and Eq. (2.49) we can reconstruct a function $\tilde{h} \in H^{2}(\bar{\Pi})$ from its boundary value function on $\mathbb{R}^{+}$. This is not quite our goal since we would like to reconstruct the function from its boundary value on the negative real axis $\mathbb{R}^{-}$. This problem, however, is solved by taking advantage of the analyticity properties of $\tilde{h}$. Closing a contour integration in the lower half-plane we obtain ( $s$ is a real number)

$$
\frac{1}{\sqrt{2 \pi}} \int_{-\infty}^{0} \tilde{h}(x) x^{-i s-\frac{1}{2}} d x+\frac{1}{\sqrt{2 \pi}} \int_{0}^{\infty} \tilde{h}(x) x^{-i s-\frac{1}{2}} d x=0
$$

and hence

$$
\tilde{E}(s)=-\frac{1}{\sqrt{2 \pi}} \int_{-\infty}^{0} \tilde{h}(x) x^{-i s-\frac{1}{2}} d x .
$$

Using Eq. (2.50) at the r.h.s. of Eq. (2.49) and taking $\phi \rightarrow 0$ we obtain

$$
\tilde{h}(x)=-\frac{1}{2 \pi} \int_{-\infty}^{\infty} d s \int_{-\infty}^{0} d x^{\prime} \tilde{h}\left(x^{\prime}\right) x^{\prime-i s-\frac{1}{2}} x^{i s-\frac{1}{2}} \quad, x>0 .
$$

Given the boundary value on $\mathbb{R}^{-}$of a function $\tilde{h} \in H^{2}(\bar{\Pi})$, Eq. (2.51) enables us to obtain its boundary value on $\mathbb{R}^{+}$. We can now construct the inverse 
map $\left(\theta^{*}\right)^{-1}$ according to the procedure described above. Given a function $h_{+} \in \theta^{*} L^{2}\left(\mathbb{R}^{+}\right)$we have for, $E \geq 0$,

$$
\begin{aligned}
& {\left[\left(\theta^{*}\right)^{-1} h_{+}\right](E)=} \\
& \quad=\left[\mathbf{P}_{\mathbb{R}^{+}} h_{+}\right](E)+\frac{1}{2 \pi} \int_{-\infty}^{\infty} d s \int_{-\infty}^{0} d \sigma\left[\mathbf{P}_{\mathbb{R}^{-}} h_{+}\right](\sigma) \sigma^{-i s-\frac{1}{2}} E^{i s-\frac{1}{2}} .
\end{aligned}
$$

This completes the proof of Lemma 2.2.

Eq. (2.39), Lemma 2.1 and Lemma 2.2 imply Eq. (2.40) and Theorem 2.5 is proved.

Theorem 2.1, Theorem 2.4 and Theorem 2.5 provide the basic nesting structure for the description of quantum mechanical resonances. The fact that for each $t \geq 0$ the evolution $\mathbf{U}(t)$ transforms, both in the incoming contractive nesting and in the outgoing contractive nesting, into elements of $\left\{T_{u(t)}\right\}_{t \geq 0}$, the semigroup of Toeplitz operators on $H^{+}(\mathbb{R})$, suggests that the Sz.-Nagy-Foias theory, providing the basic mechanism of the Lax-Phillips scattering theory, may be used in the description of resonances. In particular, the Lax-Phillips semigroup in Eq. (1.20) may be used in the description of the time evolution of quantum mechanical resonances and the relation between resonances and second sheet poles of the $S$-matrix in the lower half-plane (or first sheet zeros of the $S$-matrix in the upper half-plane) may be shown in some cases to be a result of the Sz.-Nagy-Foias theory of contractions.

The following proposition indicates on the way the Sz.-Nagy-Foias theory may be used:

Proposition $2.4\left(H^{\infty}\right.$ case) Assume that in $E q$. (2.38) $\tilde{\mathbf{S}}: L^{2}\left(\mathbb{R}^{+}\right) \mapsto$ $L^{2}\left(\mathbb{R}^{+}\right)$is the boundary value on $\mathbb{R}^{+}$of some function $\mathcal{S} \in H^{\infty}(\Pi)$. Suppose, furthermore, that $\mathcal{S}$ has a single, simple, zero at the point $z=\bar{\mu}, \operatorname{Im} \mu<0$ in $\Pi$. Let $f_{\text {in }}=\hat{\boldsymbol{\Omega}}_{-} f$ and $f_{\text {out }}=\hat{\boldsymbol{\Omega}}_{+} f$ for some $f \in \mathcal{H}_{a c}$. We have

$$
f_{\text {out }}(\sigma)=\left[\mathbf{S}_{\text {nest }} f_{\text {in }}\right](\sigma)=\frac{\sigma-\bar{\mu}}{\sigma-\mu}\left[\mathbf{S}_{\text {nest }}^{\prime} f_{\text {in }}\right](\sigma)-i 2 \operatorname{Im} \mu\left[\mathbf{P}_{-} \mathcal{S}^{\prime} f_{\text {in }}^{-}\right](\mu) \frac{1}{\sigma-\mu}
$$

where $f_{\text {in }}^{-} \in H^{-}(\mathbb{R})$ is such that $\mathbf{P}_{\mathbb{R}^{-}}\left(f_{\text {in }}+f_{\text {in }}^{-}\right)=0, \mathbf{S}_{\text {nest }}^{\prime}=\theta^{*} \tilde{\mathcal{S}}^{\prime}\left(\theta^{*}\right)^{-1}, \tilde{\mathcal{S}}^{\prime}$ is the boundary value on $\mathbb{R}^{+}$of a function $\mathcal{S}^{\prime} \in H^{\infty}(\Pi)$ and $\mathcal{S}^{\prime}$ has no zeros in $\Pi$.

Proof: 
We need first to consider the structure theory of $H^{p}$ functions on the upper half-plane [Hof, D]. We have the following definition and subsequent theorem

Definition (Blaschke product): A Blaschke product on the upper half-plane is an analytic function $b(z)$ on $\Pi$ of the form

$$
b(z)=\left(\frac{z-i}{z+i}\right)^{m} \prod_{n} \frac{\left|z_{n}^{2}+1\right|}{z_{n}^{2}+1} \cdot \frac{z-z_{n}}{z-\bar{z}_{n}}
$$

here $m$ in a nonnegative integer and $z_{n}, \operatorname{Im} z>0$, are zeros of $b(\cdot)$ in $\Pi$, finite or infinite in number.

Theorem 2.6 If $f \in H^{p}(\Pi)(0<p \leq \infty)$ and $f \not \equiv 0$, then $f(\cdot)=b(\cdot) g(\cdot)$, where $g(\cdot)$ is a non-vanishing $H^{p}(\Pi)$ function. The boundary value function of $f(\cdot)$ on $\mathbb{R}$ satisfies $|g(x)|=|f(x)|$ a.e. $(x \in \mathbb{R})$. The function $b(\cdot)$ is a Blaschke product of the form given in Eq. (2.54) and $z_{n}$ are the zeros $\left(z_{n} \neq i\right)$ of $f$ in $\Pi$.

By the definition of a Blaschke product and Theorem 2.6, if the function $\mathcal{S} \in H^{\infty}(\Pi)$ has only a single zero in $\Pi$ then it must be of the form

$$
\mathcal{S}(z)=\frac{z-\bar{\mu}}{z-\mu} \mathcal{S}^{\prime}(z) \quad \operatorname{Im} z>0, \operatorname{Im} \mu<0
$$

where $\mathcal{S}^{\prime} \in H^{\infty}(\Pi)$ has no zeros in $\Pi$. By assumption $\tilde{\mathbf{S}}$ is the boundary function of $\mathcal{S}$ on $\mathbb{R}^{+}$and so

$$
\tilde{\mathbf{S}}(E)=\frac{E-\bar{\mu}}{E-\mu} \mathcal{S}^{\prime}(E), \quad E \geq 0 .
$$

Inserting this form of $\tilde{\mathbf{S}}$ into Eq. (2.40) we get

$$
\begin{aligned}
& {\left[\mathbf{S}_{\text {nest }} f_{\text {in }}\right](\sigma)=\frac{1}{2 \pi} \int_{0}^{\infty} d E \frac{i}{\sigma-E+i 0^{+}} \frac{E-\bar{\mu}}{E-\mu} \mathcal{S}^{\prime}(E)\left[\mathbf{P}_{\mathbb{R}^{+}} f_{i n}\right](\sigma)+\frac{1}{4 \pi^{2}} \times} \\
& \int_{0}^{\infty} d E \frac{i}{\sigma-E+i 0^{+}} \frac{E-\bar{\mu}}{E-\mu} \mathcal{S}^{\prime}(E) \int_{-\infty}^{\infty} d s \int_{-\infty}^{0} d \sigma^{\prime}\left[\mathbf{P}_{\mathbb{R}^{-}} f_{i n}\right]\left(\sigma^{\prime}\right) \sigma^{-i s-\frac{1}{2}} E^{i s-\frac{1}{2}}
\end{aligned}
$$

in the first integral here we can change the integration interval to the negative real axis by using a contour integration in the upper half-plane (we 
recall that $H^{\infty}(\Pi)$ can be regarded as an algebra on $\left.H^{2}(\Pi)\right)$. We get

$$
\begin{aligned}
& \frac{1}{2 \pi} \int_{0}^{\infty} d E \frac{i}{\sigma-E+i 0^{+}} \frac{E-\bar{\mu}}{E-\mu} \mathcal{S}^{\prime}(E)\left[\mathbf{P}_{\mathbb{R}^{+}} f_{i n}\right](\sigma)= \\
= & \frac{\sigma-\bar{\mu}}{\sigma-\mu} \mathcal{S}^{\prime}(\sigma) f_{i n}(\sigma)-\frac{1}{2 \pi} \int_{-\infty}^{0} d E \frac{i}{\sigma-E+i 0^{+}} \frac{E-\bar{\mu}}{E-\mu} \mathcal{S}^{\prime}(E)\left[\mathbf{P}_{\mathbb{R}^{-}} f_{i n}\right](E) .
\end{aligned}
$$

Since $f_{i n} \in \hat{\mathbf{\Omega}}^{-} \mathcal{H}_{a c}$ then it is in the range of $\theta^{*}$. Hence (see the proof of Theorem 2.5) there is some function $f_{i n}^{-} \in H^{2}(\bar{\Pi})$ such that $\mathbf{P}_{\mathbb{R}^{-}}\left(f_{\text {in }}+f_{\text {in }}^{-}\right)=$ 0. Using Eq. (2.51) we obtain

$$
\left[\mathbf{P}_{\mathbb{R}^{+}} f_{i n}^{-}\right](E)=\frac{1}{2 \pi} \int_{-\infty}^{+\infty} d s \int_{-\infty}^{0} d \sigma^{\prime}\left[\mathbf{P}_{\mathbb{R}^{-}} f_{i n}\right]\left(\sigma^{\prime}\right) \sigma^{\prime-i s-\frac{1}{2}} E^{i s-\frac{1}{2}} \quad, E \geq 0 .
$$

Thus we get

$$
\begin{aligned}
{\left[\mathbf{S}_{\text {nest }} f_{i n}\right](\sigma)=\frac{\sigma-\bar{\mu}}{\sigma-\mu} \mathcal{S}^{\prime}(\sigma) f_{i n}(\sigma) } & \\
& \quad+\frac{1}{2 \pi} \int_{-\infty}^{\infty} d E \frac{i}{\sigma-E+i 0^{+}} \frac{E-\bar{\mu}}{E-\mu} \mathcal{S}^{\prime}(E) f_{i n}^{-}(E)
\end{aligned}
$$

For the second term on the r.h.s. of Eq. (2.59) we have the following decomposition

$$
\begin{aligned}
\frac{1}{2 \pi} \int_{-\infty}^{\infty} d E \frac{i}{\sigma-E+i 0^{+}} \frac{E-\bar{\mu}}{E-\mu} \mathcal{S}^{\prime}(E) f_{i n}^{-}(E)= \\
=\frac{1}{2 \pi} \int_{-\infty}^{\infty} d E \frac{i}{\sigma-E+i 0^{+}} \frac{E-\bar{\mu}}{E-\mu}\left[\left(\mathbf{P}_{+}+\mathbf{P}_{-}\right) \mathcal{S}^{\prime} f_{i n}^{-}\right](E)= \\
\quad=\frac{\sigma-\bar{\mu}}{\sigma-\mu}\left[\mathbf{P}_{+} \mathcal{S}^{\prime} f_{i n}^{-}\right](\sigma)-i 2 \operatorname{Im} \mu\left[\mathbf{P}_{-} \mathcal{S}^{\prime} f_{i n}^{-}\right](\mu) \frac{1}{\sigma-\mu}
\end{aligned}
$$

The second equality on the r.h.s. of Eq. (2.60) is obtained by making use of the properties of the projections $\mathbf{P}_{+}$and $\mathbf{P}_{-}$is order to close contour integrals in the upper, respectively lower half-plane. Taking notice of the fact that $\mathcal{S}^{\prime}$ is in $H^{\infty}(\Pi)$ and hence $\mathcal{S}^{\prime} f_{\text {in }} \in H^{+}(\mathbb{R})$ we finally obtain

$$
\left[\mathbf{S}_{\text {nest }} f_{i n}\right](\sigma)=\frac{\sigma-\bar{\mu}}{\sigma-\mu}\left[\mathbf{P}_{+} \mathcal{S}^{\prime}\left(f_{i n}+f_{i n}^{-}\right)\right](\sigma)-i 2 \operatorname{Im} \mu\left[\mathbf{P}_{-} \mathcal{S}^{\prime} f_{i n}^{-}\right](\mu) \frac{1}{\sigma-\mu} .
$$


Moreover, from the procedure for the construction of $\left(\theta^{*}\right)^{-1}$ it follows that $f_{i n}+f_{i n}^{-}=\mathbf{I}\left(\theta^{*}\right)^{-1} f_{i n}$, so that $\mathbf{P}_{+} \mathcal{S}^{\prime}\left(f_{i n}+f_{i n}^{-}\right)=\mathbf{P}_{+} \mathcal{S}^{\prime} \mathbf{I}\left(\theta^{*}\right)^{-1} f_{i n}=$ $\mathbf{P}_{+} \mathbf{I} \tilde{\mathcal{S}}^{\prime}\left(\theta^{*}\right)^{-1} f_{\text {in }}=\theta^{*} \tilde{\mathcal{S}}^{\prime}\left(\theta^{*}\right)^{-1} f_{\text {in }}$ and the proof of Proposition 2.4 is complete.

The Blaschke factor $B_{\mu}$ defined by (see Eq. (2.54))

$$
B_{\mu}(z)=\frac{z-\bar{\mu}}{z-\mu}
$$

is an inner function, hence we can define the subspace $\hat{\mathcal{K}}_{\mu} \subset H^{+}(\mathbb{R})$ by

$$
\hat{\mathcal{K}}_{\mu} \equiv H^{+}(\mathbb{R}) \ominus B_{\mu} H^{+}(\mathbb{R})
$$

and the Lax-Phillips semigroup $\{\hat{\mathbf{Z}}(t)\}_{t \in \mathbb{R}^{+}}$according to Eq. (1.20), i.e.,

$$
\hat{\mathbf{Z}}(t)=T_{u(t)} \mid \hat{\mathcal{K}}_{\mu} .
$$

The dimension of the subspace $\hat{\mathcal{K}}_{\mu}$ is one and, up to a multiplicative constant, the eigenvector $x_{\mu} \in \hat{\mathcal{K}}_{\mu}$ of $\hat{\mathbf{Z}}(t)$ is given by

$$
x_{\mu}(\sigma)=\frac{1}{\sigma-\mu} .
$$

Eq. (2.53) can be written then in the form

$$
f_{\text {out }}=B_{\mu} \mathbf{S}_{\text {nest }}^{\prime} f_{\text {in }}-i 2 \operatorname{Im} \mu\left[\mathbf{P}_{-} \mathcal{S}^{\prime} f_{\text {in }}^{-}\right](\mu) x_{\mu}
$$

and, using Eq. (2.5) we have, for $t \geq 0$,

$$
\begin{array}{r}
\hat{\mathbf{\Omega}}_{+} \mathbf{U}(t) f=T_{u(t)} f_{\text {out }}=T_{u(t)} B_{\mu} \mathbf{S}_{\text {nest }}^{\prime} f_{\text {in }}-i\left(2 \operatorname{Im} \mu\left[\mathbf{P}_{-} \mathcal{S}^{\prime} f_{\text {in }}^{-}\right](\mu)\right) \hat{\mathbf{Z}}(t) x_{\mu}= \\
=T_{u(t)} B_{\mu} \mathbf{S}_{\text {nest }}^{\prime} f_{\text {in }}-i\left(2 \operatorname{Im} \mu\left[\mathbf{P}_{-} \mathcal{S}^{\prime} f_{\text {in }}^{-}\right](\mu)\right) e^{-i \mu t} x_{\mu} . \quad(2.67)
\end{array}
$$

Eq. (2.67) can be interpreted as implying that in the outgoing nesting "representation" the zero of the $S$-matrix in the upper half-plane corresponds, through the mechanism of the Sz.-Nagy-Foias theory described in Section 1, to a resonance for which the characteristic, exponentialy decaying, evolution in time is given in terms of the action of a Lax-Phillips type semigroup. The resonance state is identified with the eigenvector $x_{\mu}$ of this semigroup. Eq. (2.67) clearly exhibits the decomposition, in the nesting space $H^{+}(\mathbb{R})$, of the state $f_{\text {out }}=\hat{\boldsymbol{\Omega}}_{+} f$ corresponding to an element $f \in \mathcal{H}_{a c}$, into a resonance 
part and a contribution coming from the subspace in $H^{+}(\mathbb{R})$ orthogonal to the resonance.

Define

$$
\Lambda_{\hat{\mathbf{\Omega}}_{+}}=\hat{\mathbf{\Omega}}_{+}^{*} \hat{\mathbf{\Omega}}_{+} \mathcal{H}_{a c} \subset \mathcal{H}_{a c} .
$$

The set $\Lambda_{\hat{\Omega}_{+}}$is a linear space in $\mathcal{H}_{a c}$ which is dense in $\mathcal{H}_{a c}$. For an element $g$ in $\Lambda_{\hat{\boldsymbol{\Omega}}_{+}}$there is a unique element $h \in \mathcal{H}_{a c}$ such that $g=\hat{\boldsymbol{\Omega}}_{+}^{*} \hat{\boldsymbol{\Omega}}_{+} h$. Then, for $t \geq 0$ and $f, g \in \Lambda_{\hat{\mathbf{\Omega}}_{+}}$we have

$$
\begin{aligned}
& \quad(g, \mathbf{U}(t) f)_{\mathcal{H}_{\text {ac }}}=\left(\hat{\boldsymbol{\Omega}}_{+}^{*} \hat{\boldsymbol{\Omega}}_{+} h, \mathbf{U}(t) f\right)_{\mathcal{H}_{\text {ac }}}=\left(\hat{\boldsymbol{\Omega}}_{+} h, \hat{\boldsymbol{\Omega}}_{+} \mathbf{U}(t) f\right)_{H^{+}(\mathbb{R})}= \\
& =\left(h_{\text {out }}, T_{u(t)} f_{\text {out }}\right)_{H^{+}(\mathbb{R})}= \\
& =\left(h_{\text {out }}, u(t) B_{\mu} \mathbf{S}_{\text {nest }}^{\prime} f_{\text {in }}\right)_{H^{+}(\mathbb{R})}-i 2 \operatorname{Im} \mu\left[\mathbf{P}_{-} \mathcal{S}^{\prime} f_{\text {in }}^{-}\right](\mu)\left(h_{\text {out }}, \hat{\mathbf{Z}}(t) x_{\mu}\right)_{H^{+}(\mathbb{R})}= \\
& =\left(h_{\text {out }}, u(t) B_{\mu} \mathbf{S}_{\text {nest }}^{\prime} f_{\text {in }}\right)_{H^{+}(\mathbb{R})}-i 2 \operatorname{Im} \mu\left[\mathbf{P}_{-} \mathcal{S}^{\prime} f_{\text {in }}^{-}\right](\mu) e^{-i \mu t}\left(h_{\text {out }}, x_{\mu}\right)_{H^{+}(\mathbb{R})}
\end{aligned}
$$

where $g=\hat{\mathbf{\Omega}}_{+}^{*} \hat{\mathbf{\Omega}}_{+} h, h_{\text {out }}=\hat{\boldsymbol{\Omega}}_{+} h$. and $u(t)$ is the unitary group defined in Eq. (1.14). Thus, on a dense set in $\mathcal{H}_{a c}$, matrix elements of the evolution can be calculated in the outgoing contractive nesting. The example of an $H^{\infty} S$-matrix gives a clear demonstration for the possible use of the Sz.Nagy-Foias theory of contractions in the framework of quantum mechanical scattering problems.

\section{Comments - The contractive containment of Hilbert spaces}

Consider the following definition of a bounded and contractive containment of Hilbert space ([Sar]):

Definition (bounded and contractive containment): If $\mathcal{H}_{0}$ is a Hilbert space, one says that another Hilbert space $\mathcal{H}_{1}$ is contained boundedly in $\mathcal{H}_{0}$ if it is a vector subspace of $\mathcal{H}_{0}$ and if the inclusion map of it into $\mathcal{H}_{0}$ is bounded. If the inclusion map is a contraction, one says that $\mathcal{H}_{1}$ is contained contractively in $\mathcal{H}_{0}$.

If $\mathbf{A}$ is a bounded operator from the Hilbert space $\mathcal{H}_{1}$ into the Hilbert space $\mathcal{H}_{0}$, then we define $\mathcal{M}(\mathbf{A}) \subset \mathcal{H}_{0}$ to be the range of $\mathbf{A}$ with the Hilbert space structure that makes $\mathbf{A}$ a coisometry from $\mathcal{H}_{1}$ onto $\mathcal{M}(\mathbf{A})$. Thus if $x$ and $y$ are vectors in $\mathcal{H}_{1}$ and if they are orthogonal to the kernel of $\mathbf{A}$ then

$$
(\mathbf{A} x, \mathbf{A} y)_{\mathcal{M}(\mathbf{A})}=(x, y)_{\mathcal{H}_{1}} .
$$


The space $\mathcal{M}(\mathbf{A})$ is contained boundedly in $\mathcal{H}_{0}$, and if $\mathbf{A}$ is a contraction from $\mathcal{H}_{1}$ into $\mathcal{H}_{0}$ then $\mathcal{M}(\mathbf{A})$ is contained contractively in $\mathcal{H}_{0}$. Every Hilbert space contained boundedly in $\mathcal{H}_{0}$ is such an operator range, since it is the range of the inclusion map of it into $\mathcal{H}_{0}$.

Another important notion is that of a complementary space [Sar]. If $\mathbf{A}$ : $\mathcal{H}_{1} \mapsto \mathcal{H}_{0}$ is a Hilbert space contraction, then $\mathcal{H}(\mathbf{A}) \equiv \mathcal{M}\left(\left(1-\mathbf{A} \mathbf{A}^{*}\right)^{1 / 2}\right) \subset$ $\mathcal{H}_{0}$ is called the complementary space of $\mathcal{M}(\mathbf{A})$. If $\mathcal{M}(\mathbf{A})$ is an ordinary subspace, that is, if $\mathbf{A}$ is a partial isometry, then $\mathbf{A} \mathbf{A}^{*}$ and $1-\mathbf{A} \mathbf{A}^{*}$ are complementary orthogonal projections, and $\mathcal{H}(\mathbf{A})$ is the ordinary orthogonal complement of $\mathcal{M}(\mathbf{A})$. If $\mathbf{A}$ is not a partial isometry then the intersection $\mathcal{M}(\mathbf{A}) \cap \mathcal{H}(\mathbf{A})$, called the overlapping space [Sar], is non-trivial. In this case we have

$$
\mathcal{M}(\mathbf{A}) \cap \mathcal{H}(\mathbf{A})=\mathbf{A} \mathcal{H}\left(\mathbf{A}^{*}\right)
$$

In addition we have

$$
\mathcal{H}_{0}=\mathcal{H}(\mathbf{A})+\mathcal{M}(\mathbf{A})
$$

where is Eq. (3.3) vectors in $\mathcal{M}(\mathbf{A})$ and $\mathcal{H}(\mathbf{A})$ are considered as elements of $\mathcal{H}_{0}$.

Applying the definition above and the subsequent discussion to the nesting structure $\left(\mathcal{H}_{a c}, H^{+}(\mathbb{R}), \hat{\boldsymbol{\Omega}}_{+}\right)$it is clear that $\mathcal{M}\left(\hat{\boldsymbol{\Omega}}_{+}\right) \subset H^{+}(\mathbb{R})$ is contractively contained in $H^{+}(\mathbb{R})$ (of course, the same statement is valid for $\left.\mathcal{M}\left(\hat{\boldsymbol{\Omega}}_{-}\right) \subset H^{+}(\mathbb{R})\right)$. Moreover, since $\hat{\boldsymbol{\Omega}}_{+}: \mathcal{H}_{a c} \mapsto H^{+}(\mathbb{R})$ is injective, the scalar product in $\mathcal{M}\left(\hat{\boldsymbol{\Omega}}_{+}\right)$defined in Eq. (3.1) can be written in the form

$$
(x, y)_{\mathcal{M}\left(\hat{\boldsymbol{\Omega}}_{+}\right)} \equiv\left(\hat{\boldsymbol{\Omega}}_{+}^{-1} x, \hat{\mathbf{\Omega}}_{+}^{-1} y\right)_{\mathcal{H}_{a c}} .
$$

Regarding $\hat{\boldsymbol{\Omega}}_{+}$as a map from $\mathcal{H}_{a c}$ onto $\mathcal{M}\left(\hat{\boldsymbol{\Omega}}_{+}\right)$, equipped with the scalar product defined in Eq. (3.4), we find that it is unitary and $\mathcal{M}\left(\hat{\boldsymbol{\Omega}}_{+}\right)$is a representation of $\mathcal{H}_{a c}$ (in terms of functions belonging to $H^{+}(\mathbb{R})$ ). Since $\hat{\boldsymbol{\Omega}}_{+}$ is a contraction we can construct the complementary space $\mathcal{H}\left(\hat{\boldsymbol{\Omega}}_{+}\right) \subset H^{+}(\mathbb{R})$ by

$$
\mathcal{H}\left(\hat{\boldsymbol{\Omega}}_{+}\right)=\mathcal{M}\left(\left(1-\hat{\boldsymbol{\Omega}}_{+} \hat{\mathbf{\Omega}}_{+}^{*}\right)^{1 / 2}\right)
$$

and Eq. (3.2) and Eq. (3.3) imply that

$$
\mathcal{M}\left(\hat{\boldsymbol{\Omega}}_{+}\right) \cap \mathcal{H}\left(\hat{\boldsymbol{\Omega}}_{+}\right)=\hat{\boldsymbol{\Omega}}_{+} \mathcal{H}\left(\hat{\mathbf{\Omega}}_{+}^{*}\right) \neq\{0\}
$$

and

$$
H^{+}(\mathbb{R})=\mathcal{H}\left(\hat{\boldsymbol{\Omega}}_{+}\right)+\mathcal{M}\left(\hat{\boldsymbol{\Omega}}_{+}\right)
$$


We know that $\hat{\boldsymbol{\Omega}}_{+} \mathcal{H}_{a c}$ is dense in $H^{+}(\mathbb{R})$, hence for any $f \in H^{+}(\mathbb{R})$ there exists some sequence of elements $\left\{f_{n}\right\}_{n \in N}, f_{n} \in \mathcal{H}_{a c}$ such that

$$
\lim _{n \rightarrow \infty}\left\|f-\hat{\mathbf{\Omega}}_{+} f_{n}\right\|_{H^{+}(\mathbb{R})}=0 .
$$

Observe that by the one to one correspondence between $\mathcal{H}_{a c}$ and $\mathcal{M}\left(\hat{\boldsymbol{\Omega}}_{+}\right)$, the continuity of $\hat{\boldsymbol{\Omega}}_{+}$and its contractive property, if a sequence $\left\{f_{n}\right\}_{n \in N}$ of elements $f_{n} \in \mathcal{H}_{a c}$ converges to an element $f \in \mathcal{H}_{a c}$ we have

$$
\lim _{n \rightarrow \infty}\left\|\hat{\boldsymbol{\Omega}}_{+} f-\hat{\boldsymbol{\Omega}}_{+} f_{n}\right\|_{H^{+}(\mathbb{R})} \leq \lim _{n \rightarrow \infty}\left\|f-f_{n}\right\|_{\mathcal{M}\left(\hat{\boldsymbol{\Omega}}_{+}\right)}=\lim _{n \rightarrow \infty}\left\|f-f_{n}\right\|_{\mathcal{H}_{a c}}=0 .
$$

Hence all converging sequences in $\mathcal{H}_{a c}$ correspond, under the mapping $\hat{\boldsymbol{\Omega}}_{+}$, to sequences in $H^{+}(\mathbb{R})$ converging to elements in $\mathcal{M}\left(\hat{\boldsymbol{\Omega}}_{+}\right)$in the topology of the space $H^{+}(\mathbb{R})$. Conversly, any element in $\mathcal{M}\left(\hat{\boldsymbol{\Omega}}_{+}\right)$, regarded as a subset of $H^{+}(\mathbb{R})$, corresponds (under the mapping by $\hat{\boldsymbol{\Omega}}_{+}$) to some sequence converging in $\mathcal{H}_{a c}$. Thus elements in $\mathcal{M}\left(\hat{\boldsymbol{\Omega}}_{+}\right)$are associated with the existence of converging sequences in $\mathcal{H}_{a c}$. We conclude that elements in $H^{+}(\mathbb{R})$ that do not correspond (under the mapping by $\hat{\boldsymbol{\Omega}}_{+}$) to any converging sequence in $\mathcal{H}_{a c}$ are all in $\mathcal{H}\left(\hat{\boldsymbol{\Omega}}_{+}\right) \backslash \mathcal{M}\left(\hat{\boldsymbol{\Omega}}_{+}\right)$. In particular, resonances, which are not elements of $\mathcal{H}_{a c}$ and do not correspond to any converging sequence in $\mathcal{H}_{a c}$, always belong to the complementary space $\mathcal{H}\left(\hat{\boldsymbol{\Omega}}_{+}\right)$. In the discussion above of an $S$-matrix associated with an $H^{\infty}(\Pi)$ function this observation implies that the subspace $\hat{\mathcal{K}}_{\mu}$ containing the resonance has the property that $\hat{\mathcal{K}}_{\mu} \subset \mathcal{H}\left(\hat{\boldsymbol{\Omega}}_{+}\right) \backslash \hat{\boldsymbol{\Omega}}_{+} \mathcal{H}_{a c}$.

\section{Conclusions}

This paper introduces an application of the Sz.-Nagy-Foias theory of contraction operators on Hilbert space for the description of resonances in quantum mechanical scattering problems for which the generator of evolution is bounded from below. This approach is based on the construction, in Section 2 above, of a framework analogous to that of the Lax-Phillips scattering theory. Thus, in this framework there exists an outgoing nesting structure, an incoming nesting structure and a nested $S$-matrix, which are analogous to the Lax-Phillips translation representations and the Lax-Phillips $S$-matrix respectively. In the outgoing and incoming nesting structures the evolution is transformed, for times $t \geq 0$ into a continuous semigroup of Toeplitz operators. It was shown that, in the case that the original $S$-matrix is a bounded analytic function in the upper half-plane, its zeros in the upper half-plane (or poles of its analytic continuation to the lower half-plane) correspond 
to the existence of a subspace of the nesting space such that the (nested) evolution restricted to this subspace defines a Lax-Phillips type semigroup providing the typical exponential decay behavior of the resonance. The relation between the zeros of the $S$-matrix and the eigenvalues of the generator of the semigroup are seen to be a result of the Sz.-Nagy-Foias theory of contractions. One may note, furthermore, that the two conditions for an appropriate description of a resonance, set in Section 1, are formaly satisfied. However, the interpretation of the resonance state (the eigenvector $x_{\mu}$ of the generator of the Lax-Phillips type semigroup $\{\hat{\mathbf{Z}}(t)\}_{t \geq 0}$ appearing in Eq. (2.68)) merits some additional investigation.

The results presented in this work are limited in two obvious ways. The first is the fact that the nesting structures are constructed for scalar valued functions and the second stems from the fact that the appearance of the Lax-Phillips semigroup was shown under the assumption that the $S$-matrix is the boundary value function on $\mathbb{R}^{+}$of an $H^{\infty}$ function in the upper halfplane. An extension of the nesting framework to the case of an absolutely continuous spectrum of $\mathbf{H}_{0}$ and $\mathbf{H}$ with finite multiplicity seem to follow in a straightforward way from the framework developed here. More general cases may present additional difficulties.

Concerning the second difficulty raised above, it should be emphasized that the general framework developed in Section 2, upto and including Theorem 2.5, exist regardless of any particular assumptions on the properties of the original $S$-matrix. More elaborate tools from operator theory may be invoked if more general assumptions, other than the simplifying assumption of an $H^{\infty} S$-matrix, are made. Possible extensions of the framework presented in this paper, along these lines, will be investigated elsewhere.

\section{Acknowledgments}

The author wishes to thank L.P. Horwitz and Paul A. Fuhrmann for useful discussions.

\section{References}

[A] Agmon S.: A perturbation theory for resonances. Comm. Pure, Appl. Math. 51 1255-1309 (1998); DOI 10.1002/(SICI)1097-0312 $(199811 / 12) 51$.

[AC] Aguilar J. and Combes J.M.: A class of analytic perturbations for one-body Schrödinger Hamiltonians. Comm. Math. Phys. 22 269-279 (1971). 
[B] Baumgartel W.: Partial resolvent and spectral concentration. Math. Nachr. 69 107-121 (1975).

[BC] Balslev E. and Combes J.M.: Spectral properties of many-body Schrödinger operators with dilatation-analytic interactions. Comm. Math. Phys. 22 280-294 (1971).

[BG] Bohm A. and Gadella M.: Dirac Kets, Gamow Vectors and Gel'fand Triplets, Lecture notes in Physics, Vol 348, Springer-Verlag, Berlin (1989).

[BS] Bailey T. and Schieve W.C.: Complex energy eigenstates in quantum decay models.. Nuovo Cimento 47A 231-250 (1978).

[CFS] Cornfield I.P. and Formin S.V. and Sinai Ja. G.: Ergodic Theory, Springer-Verlag, Berlin (1982).

[CS] Costin O. and Soffer A.: Resonance theory for Schrödinger operators, Dedicated to Joel L. Lebowitz. Comm. Math. Phys. 224 133-152 (2001); DOI 10.1007/s002200100558.

[D] Duren P.L. :Theory of $H^{p}$ spaces, Academic Press, New York and London (1970).

[EH] Eisenberg E. and Horwitz L.P.: Time, irreversibility, and unstable systems in quantum physics, in Advances in Chemical Physics, edited by I. Prigogine and S. Rice, Vol. XCIX, Wiley, New York, pp. 245-297 (1997).

[FP] Flesia C. and Piron C.: La théorie de la diffusion de Lax-Phillips dans le cas quantique. Helv. Phys. Acta 57, 697-703 (1984).

[FS] Fourès Y. and Segal I.E.: Causality and analyticity. Trans. Amer. Math. Soc. 78, 385-405 (1955).

[G] Grossmann A.: Nested Hilbert space in quantum mechanics, I. J. Math. Phys. 5, 1025-1037 (1964).

[HeSj] Helffer B. and Sjöstrand J.: Résonances en limite semi-classique. Mém. Soc. Math. France (N.S.) No. 24-25 (1986).

[HiSi] Hislop P.D. and Sigal I.M.: Introduction to Spectral Theory: with Applications to Schrödinger operators, Springer-Verlag, New-York (1996). 
[HM] Horwitz L.P. and Mizrachi L.: On the orthogonality of $K^{0}$ meson nonleptonic weak-decay residues. Nuovo Cimento. 21A 625-650 (1974).

[Hof] Hoffman K.: Banach Spaces of Analytic functions, Prentice Hall, Englewood Cliffs, N.J. (1962).

[HoSi] Horwitz L.P. ans Sigal I.M.: On a mathematical model for nonstationary physical systems. Helv. Phys. Acta 51 685-715 (1978).

[HP] Horwitz L.P. and Piron C.: The unstable system and irreversible motion in quantum theory. Helv. Phys. Acta 66, No. 7-8, 693-711 (1993).

[Hun] Hunziker W.: Distortion analyticity and molecular resonance curves. Ann. Inst. H. Poincaré Phys. Théor. 45 339-358 (1986).

[K] Kubrusly C.S.: An Introduction to Models and Decompositions in Operator Theory, Birkhauser, Boston (1997).

[LP] Lax P.D. and Phillips R.S.: Scattering Theory, Academic Press, New York (1967).

[MS] Merkli M. and Sigal I.M.: A time-dependent theory of quantum resonance, Comm. Math. Phys. 201 549-576 (1999); DOI $10.1007 / \mathrm{s} 002200050568$.

[NF] Sz.-Nagy B. and Foias C.: Harmonic Analysis of Operators on Hilbert space, North Holland Publishing Company, Amsterdam and London (1970).

[NSZ] Nakamura S. and Stefanov P. and Zworski M.: Resonance expansions of propagators in the presence of potential barriers. J. Funct. Anal. 205 180-205 (2003); DOI 10.1016/S0022-1236(02)00112-X.

[PGS] Parravincini G. and Gorini V. and Sudrashan E.C.G.: Resonances, scattering theory, and rigged Hilbert spaces. J. Math. Phys. 21 22082226 (1980).

[PV] Petkov V. and Vodev G.: Upper bounds on the number of scattering poles and the Lax-Phillips conjecture. Asymp. Anal. 7 87-104 (1993).

[PW] Paley R.E.A.C and Wiener N.: Fourier transforms in the complex domain. Amer. Math. Soc. Colloq. Pub., Vol. 19, New York (1934). 
[RR] Rosenblum M. and Rovnyak J.: Hardy Classes and Operator Theory, Oxford University Press, New York (1985).

[Sar] Sarason D.: Sub-Hardy Hilbert Space in the Unit Disc, University of Arkansas lecture notes in the mathematical sciences, Vol. 10, J. Wiley \& Sons, New-York (1994).

[SHE] Strauss Y. and Horwitz L.P. and Eisenberg E.: Representation of quantum mechanical resonances in Lax-Phillips Hilbert space. J. Math. Phys 41, No. 12, 8050-8071 (2000); DOI 10.1063/1.1310359.

[SH1] Strauss Y. and Horwitz L.P.: Representation of the resonance of a relativistic quantum field theoretical Lee-Friedrichs model in LaxPhillips scattering theory. Found. Phys. 30, No. 5, 653-694 (2000); DOI 10.1023/A:1003780726075.

[SH2] Strauss Y. and Horwitz L.P.: Lax-Phillips scattering theory of a relativistic quantum field theoretical Lee-Friedrichs model and LeeOehme-Yang-We phenomenology. J. Math. Phys. 43, No. 5, 23942422 (2002); DOI 10.1063/1.1461426.

[Sim1] Simon B.: The definition of molecular resonance curves by the method of exterior scaling. Phys. Lett. A 71 211-214 (1979).

[Sim2] Simon B: Resonances in $n$-body quantum systems with dilatation analytic potentials and the foundations of time dependent theory. Ann. of Math. (2) 97 247-274 (1973).

[SW] Soffer A. and Weinstein M.I.: Time dependent resonance theory. Geom. Func. Anal. 8 1086-1128 (1998); DOI 10.1007/s000390050124.

[SZ1] Sjöstrand J. and Zworski M.: Lower bounds on the number of scattering poles, II. J. Funct. Anal. 123 336-367 (1994); DOI 10.1006/jfan.1994.1092.

[SZ2] Sjöstrand J. and Zworski M.: Complex scaling and the distribution of scattering poles. J. Amer. Math. Soc. 4 729-769 (1991).

[VW] Van Winter C.: Fredholm equations on a Hilbert space of analytic functions. Trans. Amer. Math. Soc. 162 (1971), 103-139 (1971).

[Z] Zworski M.: Resonances in physics and geometry. Notices Amer. Math. Soc. 46 319-328 (1999).

E-mail: ystrauss@math.huji.ac.il 